\title{
Ionospheric feedback effects on the quasi-stationary coupling between LLBL and postnoon/evening discrete auroral arcs
}

\author{
M. M. Echim ${ }^{1, *}$, M. Roth $^{1}$, and J. De Keyser ${ }^{1}$ \\ ${ }^{1}$ BIRA-IASB, Avenue Circulaire 3, 1180 Bruxelles, Belgium \\ *also at: Institute for Space Sciences, Atomiştilor 409, 077125 Măgurele, Romania
}

Received: 26 October 2007 - Revised: 22 February 2008 - Accepted: 10 March 2008 - Published: 13 May 2008

\begin{abstract}
We discuss a model for the quasi-stationary coupling between magnetospheric sheared flows in the dusk sector and discrete auroral arcs, previously analyzed for the case of a uniform height-integrated Pedersen conductivity $\left(\Sigma_{P}\right)$. Here we introduce an ionospheric feedback as the variation of $\Sigma_{P}$ with the energy flux of precipitating magnetospheric electrons $\left(\epsilon_{\mathrm{em}}\right)$. One key-component of the model is the kinetic description of the interface between the duskward LLBL and the plasma sheet that gives the profile of $\Phi_{m}$, the magnetospheric electrostatic potential. The velocity shear in the dusk LLBL plays the role of a generator for the auroral circuit closing through Pedersen currents in the auroral ionosphere. The field-aligned current density, $j_{\|}$, and the energy flux of precipitating electrons are given by analytic functions of the field-aligned potential drop, $\Delta \Phi$, derived from standard kinetic models of the adiabatic motion of particles. The ionospheric electrostatic potential, $\Phi_{i}$ (and implicitely $\Delta \Phi)$ is determined from the current continuity equation in the ionosphere. We obtain values of $\Delta \Phi$ of the order of kilovolt and of $j_{\|}$of the order of tens of $\mu \mathrm{A} / \mathrm{m}^{2}$ in thin regions of the order of several kilometers at $200 \mathrm{~km}$ altitude. The spatial scale is significantly smaller and the peak values of $\Delta \Phi, j_{\|}$and $\epsilon_{\mathrm{em}}$ are higher than in the case of a uniform $\Sigma_{P}$. Effects on the postnoon/evening auroral arc electrodynamics due to variations of dusk LLBL and solar wind dynamic and kinetic pressure are discussed. In thin regions (of the order of kilometer) embedding the maximum of $\Delta \Phi$ we evidence a non-linear regime of the current-voltage relationship. The model predicts also that visible arcs form when the velocity shear in LLBL is above a threshold value depending on the generator and ionospheric plasma properties. Brighter arcs are obtained for increased velocity shear in the LLBL; their spatial scale remains virtually unmodified. The field-aligned potential drop tends to decrease with increasing LLBL den-
\end{abstract}

Correspondence to: M. M. Echim

(marius.echim@oma.be) sity. For higher values of the LLBL electron temperature the model gives negative field-aligned potential drops in regions adjacent to upward field-aligned currents.

Keywords. Ionosphere (Auroral ionosphere) - Magnetospheric physics (Auroral phenomena; Magnetosphereionosphere interactions)

\section{Introduction}

Discrete auroral arcs observed in the postnoon and evening sector are a rather common phenomenon. It has been suggested that auroral activity in these regions is associated with the Low Latitude Boundary Layer (LLBL), thus with direct solar wind - magnetosphere interaction (Bythrow et al., 1981; Evans, 1985; Lundin and Evans, 1985; Lundin et al., 1995). According to recent statistical analysis based on particle spectra measured by the DMSP satellites and ground based data (Newell et al., 2004, 2005) the locus of bright postnoon auroral spots covers a region that maps to the LLBL and to the Boundary Plasma Sheet (BPS) or Plasma Sheet Boundary Layer (PSBL). The electrodynamics of large scale (of the order of $100 \mathrm{~km}$ ) postnoon arcs seems to be sensitive to the IMF $\mathrm{B}_{z}$ orientation (Kozlovsky and Kangas, 2001). Moen et al. (1994) showed examples of postnoon discrete arcs with a thickness of 10-20 km and a lifetime of about $\sim 2$ to 5 min, embedded in a quasi-stable background luminosity. Moen et al. (1994) associated this type of auroral structures to magnetosheath plasma elements impulsively penetrating the LLBL (Lemaire, 1977; Lemaire and Roth, 1978) or the Kelvin-Helmholtz instability driven by the velocity shear in the LLBL (e.g. Hasegawa, 1976; Miura and Pritchett, 1982; Wei et al., 1990). A statistical analysis (Vo and Murphree, 1995) based on Viking UV images shows that the occurrence of postnoon auroral bright spots is favored by high solar wind speed, low density, and an IMF $B_{y}<0$, suggesting

Published by Copernicus Publications on behalf of the European Geosciences Union. 
that not only the IMF direction, but also the velocity shear plays an important role in triggering the dayside aurora. Velocity shears at ionospheric altitudes, related to a higher altitude "source" region, have been observed at the edges of discrete arcs (see, e.g. Vogt et al., 1999). A model of dayside aurora based on spatial Kelvin-Helmholtz modulations of the LLBL without ionospheric feedback has been proposed by Yamamoto and Ozaki (2005).

Modeling the Magnetosphere-Ionosphere (M-I) coupling aims to understand and evaluate the effects of changes in the macroscopic parameters of the magnetospheric and solar wind plasma (e.g. the shear of bulk velocity, the density and the electron temperature) on the morphology and electrodynamics of discrete auroral arcs. Models based on a kinetic treatment of adiabatic motion of particles have successfully described the large-scale structure (tens to hundreds of kilometers) of the field-aligned potential drop consistent with inverted-V spectra of precipitating electrons (Chiu and Schulz, 1978; Lyons, 1980; Chiu and Cornwall, 1980). Time-dependent MHD simulations based on the concept of anomalous, or turbulent, resistivity and ionospheric feedback through reflected Alfvén waves reproduced multiple arcs with spatial scales of the order of tens of kilometers or less (e.g. Atkinson, 1970; Sato, 1978; Lysak, 1986; Lysak and Song, 2002).

Based on convergent E-field distributions fitted to experimental data, Lyons (1981) has shown that arclike, narrower structures can be obtained with quasi-static kinetic models of M-I coupling. In a previous paper (Echim et al., 2007, hereinafter referred to as ERDK07) we have demonstrated the coupling between magnetospheric and ionospheric spatial scales and the formation of field-aligned current sheets and channels of precipitating energy connecting the auroral ionosphere with the generator. The latter is described by a selfconsistent solution derived as in kinetic models of tangential discontinuities (Roth et al., 1996). The spatial scales of the obtained auroral structures (field-aligned current sheets and auroral arcs) are of the order of kilometers. In this previous study we considered a uniform height-integrated Pedersen conductivity. Thus the role of the ionospheric feedback on the coupling was not investigated.

The energy deposited by auroral electrons and the additional ionization produced in regions associated with visible discrete auroral arcs have been evaluated by various models of auroral electron transport (see, e.g., Strickland et al., 1976; Sergienko and Ivanov, 1993; Solomon, 1993; Lummerzheim and Lilensten, 1994). These models emphasize the difference between secondary electrons produced by precipitating auroral particles and photoelectrons produced by incident solar UV radiation. The distribution of photoelectrons is much broader in space and more stable in time and contribute to a background ionospheric conductivity. Secondary electrons are localized in time and space in active auroral regions; their flux depends on the flux of precipitating energy and enhance locally the conductivity (see, e.g. Hardy et al.,1989). Empir- ical models (Harel et al., 1977; Robinson et al., 1987) give simple formulas that relate the height-integrated Pedersen and Hall conductivities to the energy flux of the precipitating electrons.

An enhanced height-integrated Pedersen conductivity due to precipitating auroral electrons provides a positive feedback to the coupling between the horizontal, Pedersen, ionospheric currents and the magnetospheric field-aligned ones. Early fluid models discussed the development of a feedback instability feeding the multiplicity of auroral arcs (Atkinson, 1970; Sato, 1978) and stressing the active role of the ionosphere in driving magnetospheric electron precipitation. Time-dependent models based on ideal MHD (Trakhtengertz and Feldstein, 1984; Lysak, 1986) have emphasized the possible role of Alfvén waves in accelerating auroral electrons. They have stressed the role of conductivity fluctuations for the formation of auroral arcs and their spatial scaling. Although one cannot describe a static parallel electric field in ideal MHD, it has been shown by one and two-fluid simulations (Lotko et al., 1987; Rönnmark and Hamrin, 2000) that after a sufficiently long simulation time the system driven by Alfvén waves relaxes to a quasi-static situation similar to the one described by kinetic models, i.e. with a stationary fieldaligned potential drop extending along the auroral flux tube. The feedback effect has been also introduced in steady-state kinetic models like the ones developed by Lyons (1980) and Chiu and Cornwall (1980), that will be briefly reviewed in the next section.

In this paper we do not take into account time-dependent effects but we treat a steady state solution of the current continuity equation in the ionosphere. Thus the ionospheric feedback is introduced via the Pedersen conductance, $\Sigma_{\mathrm{P}}$, that depends on the precipitating electron energy flux, $\epsilon_{\mathrm{em}}$, as described by Harel et al. (1977); any other model for $\Sigma_{P}$ can be also used. In the next section we briefly review the main features of the quasi-static coupling model. Then we illustrate and discuss the numerical solutions obtained with ionospheric feedback for various plasma parameters in the generator. The paper concludes with a summary and discussion.

\section{A quasi-stationary model for the coupling between sheared flows in the LLBL and the postnoon discrete auroral arcs}

Ad-hoc convergent electric fields in the magnetosphere were treated in earlier studies by Lyons $(1980,1981)$ as the basis for discrete arcs. A description of magnetospheric generators based on the tangential discontinuity (TD) model was given by Roth et al. (1993) and De Keyser et al. (1998). In this study we consider the inner edge of the low latitude boundary layer (LLBL) at the dusk flank of the magnetosphere. This interface separates a region of magnetospheric (MSPH) low density, stagnant plasma with a relatively high 


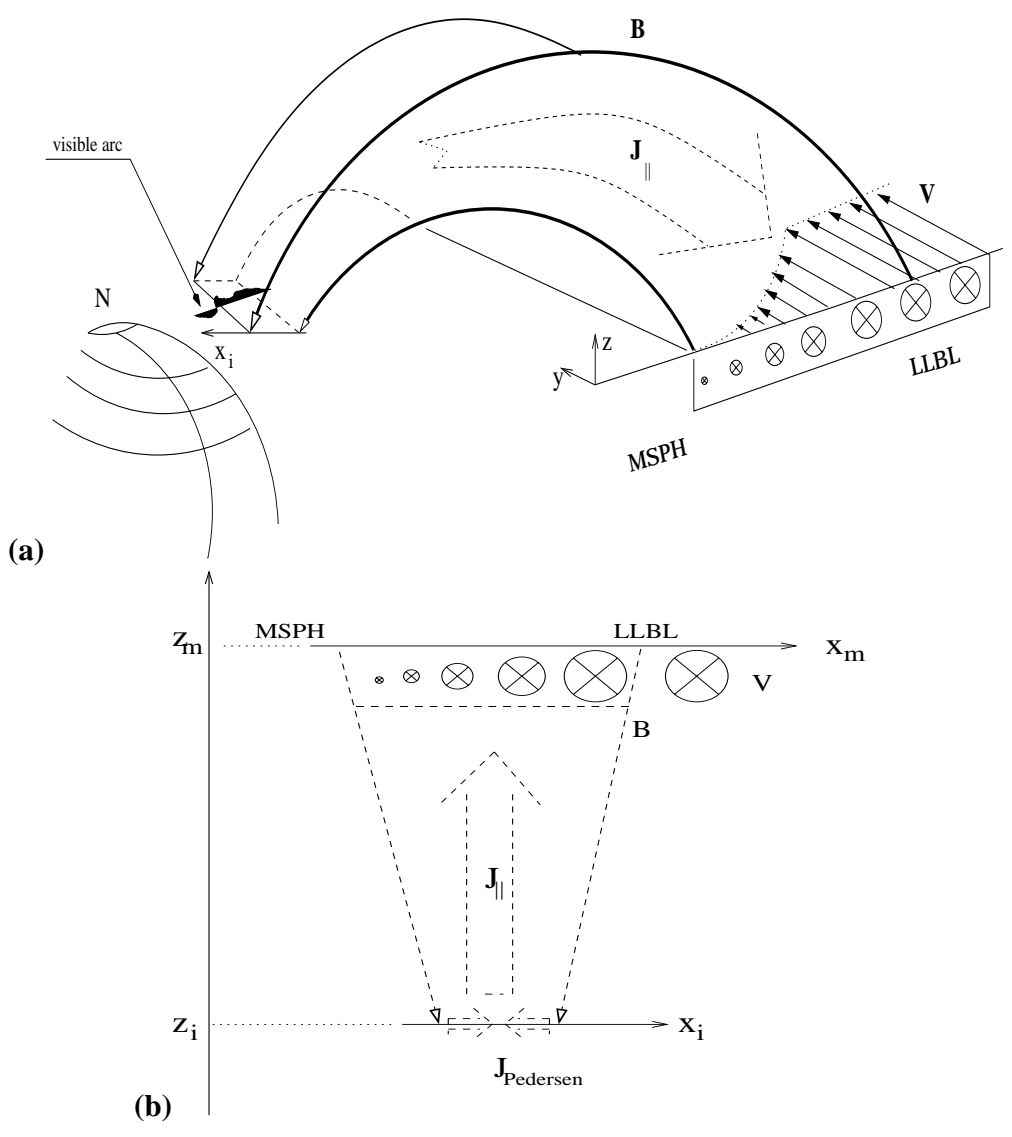

Fig. 1. Diagram illustrating the geometry considered to study the coupling between a sheared flow LLBL and the postnoon/evening ionosphere. (a) A schematic 3-D view of the magnetospheric dusk flank; (b) A simpler, conical geometry has been adopted to describe a flux tube extended from lower/ionospheric altitudes $\left(z_{i}\right)$ to upper/magnetospheric level $\left(z_{m}\right)$. The upper boundary of the flux tube coincides with a sector that includes the sheared flow layer at the interface between the LLBL and the magnetospheric dusk flank (MSPH). The sheared flow layer is described by a TD and plays the role of an auroral generator, as explained in the text. The velocity profile is illustrated by circles whose radius is proportional to the local value of the bulk velocity; the velocity is oriented anti-sunward, along the y-axis, into the page (adapted from Echim et al., 2007).

thermal energy from the adjacent denser plasma moving antisunward in the LLBL. The LLBL plasma properties (velocity, density) are closely connected to solar wind parameters. The two asymptotic states are described in Table 1. The transition between the plasma-sheet like plasma (MSPH) and the LLBL plasma is obtained numerically with a Vlasov equilibrium solution derived from the principles of kinetic models of tangential discontinuities (TD) with sheared flows, described by Roth et al. (1996). One key feature of the solution is the self-consistent electric field perpendicular to the surface of discontinuity. Note that the intensity of this convergent electric field depends on the shear of the bulk velocity, the gradient of temperature and the gradient of density.

The relative orientation of the magnetic field, plasma bulk velocity and velocity shear defining the magnetospheric plasma TD interface correspond to the inner edge of the duskward LLBL (see Fig. 1a). The solutions does not depend, however, on the chosen geometry. The model can be also applied for the dawn LLBL where divergent electric fields are likely to be obtained in the TD solution. This results in downward field-aligned currents into the ionosphere (Marklund et al., 2001). The pair of upward and downward parallel currents, respectively at the duskside and dawnside of the LLBL corresponds with the poleward region 1 parallel current (Iijima and Potemra, 1976).

The TD solution provides the profile of $\Phi_{m}$, the electrostatic potential in the magnetosphere. It describes an unloaded voltage generator like the ones discussed by Roth et al. (1993) and De Keyser et al. (1998). The duskward LLBL is coupled by magnetic field lines to the conducting ionosphere, i.e. the generator is connected to the ionospheric load. Therefore magnetospheric plasma is in contact with the ionospheric plasma. It can be assumed that the external driver sustaining the sheared plasma flow (the solar wind) do also continuously replenishes the loss cone and maintains the electric field at the inner edge of the LLBL. The effects of 
the coupling to the auroral ionosphere on the LLBL structure itself are not considered in this study. This type of effects have been discussed in the framework of fluid models of the LLBL by Lotko et al. (1987), Drakou et al. (1994), Wei et al. (1996). Lotko et al. (1987) showed that LLBL sheared flows with speeds of the order of $200 \mathrm{~km} / \mathrm{s}$ might be braked by the coupling to the ionosphere through field-aligned currents and electric field on time scales of the order of tens of minutes.

The adiabatic motion of magnetospheric and ionospheric particles originating near the LLBL sheared layer and precipitating along magnetic fields lines into the auroral ionosphere contributes to a net field-aligned current. When the Hall currents are divergence free, a situation quite often encountered in the auroral ionosphere (Sugiura, 1984), the current continuity equation in the ionosphere requires that the net parallel current in the ionosphere $\left(j_{\|}\right)$is equal to the divergence of the horizontal height-integrated Pedersen current in the ionosphere $\left(I_{P}\right)$ :

$j_{\|}\left(\Phi_{i}, \Phi_{m}\right)=-\frac{d I_{P}}{d x_{i}}=\frac{d}{d x_{i}}\left(\Sigma_{P} \frac{d \Phi_{i}}{d x_{i}}\right)$

where $x_{i}$ denotes the distance perpendicular to the arc at ionospheric altitude and $\Phi_{i}$ is the electrostatic potential in the ionosphere at altitude $z_{i}$.

The field-aligned current density, $j_{\|}$, is a function of the field-aligned potential drop, $\Delta \Phi=\Phi_{i}-\Phi_{m}$; this relationship is known as the current-voltage relation (or Ohm's law) for the auroral electric circuit. It has been computed by several authors for a $\Delta \Phi$ monotonically decreasing with altitude and assuming adiabatic motion of particles along the flux tube connecting the ionospheric load and the magnetospheric generator described by Maxwellian (Knight, 1973; Lemaire and Scherer, 1973; Chiu and Schulz, 1978), biMaxwellian (Fridman and Lemaire, 1980) or kappa (Pierrard, 1996) velocity distribution functions. A recent review on the currentvoltage relation has been published by Pierrard et al. (2007). The current-voltage relation can be linearized for a limited range of $\Delta \Phi$ (Knight, 1973; Lyons et al., 1979). In this paper we use analytical formulas for $j_{\|}(\Delta \Phi)$ obtained for a monotonically decreasing $\Delta \Phi$ and Maxwellian magnetospheric and ionospheric sources (Knight, 1973; Lemaire and Scherer, 1973). The net field-aligned current density, $j_{\|}$, is the sum of partial current densities due to magnetospheric electrons and protons as well as ionospheric electrons and three species of ionospheric ions $\left(\mathrm{H}^{+}, \mathrm{O}^{+}, \mathrm{He}^{+}\right)$. Gravitational effects are neglected. Equation (1) gives a quantitative description of the coupling between plasma and field properties at the inner edges of the LLBL (the generator), and the plasma and field properties in the ionospheric load; it is solved for the unknown $\Phi_{i}$ and the input parameters $\Phi_{m}$ and $\Sigma_{P}$.

The ionospheric feedback effect is introduced via the relationship between $\Sigma_{P}$ and the flux of precipitating en- ergy, $\epsilon_{\mathrm{em}}$, derived from the empirical formula of Harel et al. (1977):

$\Sigma_{P}=\Sigma_{P 0}+a \sqrt{\epsilon_{\mathrm{em}}}$

In Eq. (2), $\Sigma_{P}$ and $\Sigma_{P 0}$ are given in $\mathrm{S}$, and $\epsilon_{\mathrm{em}}$, in $\mathrm{Jm}^{-2} \mathrm{~s}^{-1}$. In Harel's model $\Sigma_{P 0}=0.5 \mathrm{~S}$ and $a=160 \mathrm{C}^{2} \mathrm{~s}^{3 / 2} \mathrm{~m}^{-2} \mathrm{~kg}^{-3 / 2}$. $\Sigma_{P 0}$ is the base level of the ambient conductance, produced by the solar EUV radiation. The energy flux of precipitating electrons, $\epsilon_{\mathrm{em}}$, is determined from the second-order moment of the solution of the stationary Vlasov equation (Lemaire and Scherer, 1973; Lundin and Sandahl, 1978):

$$
\begin{aligned}
\epsilon_{\mathrm{em}}= & N_{\mathrm{m}}^{-}\left(k T_{\mathrm{m}}^{-}\right) b \sqrt{\frac{k T_{\mathrm{m}}^{-}}{2 \pi m^{-}}} \times \\
& \left\{\left(2+\Delta \Phi^{*}\right)-\left[\Delta \Phi^{*}+2\left(1-\frac{1}{b}\right)\right] e^{-\frac{\Delta \Phi^{*}}{b-1}}\right\}
\end{aligned}
$$

where $N_{\mathrm{m}}^{-}$and $T_{\mathrm{m}}^{-}$are the density and temperature of the magnetospheric electrons, $b=B_{i} / B_{m}>1$ is the magnetic compression ratio and $\Delta \Phi^{*}=e \Delta \Phi / k T_{\mathrm{m}}^{-}$is the normalized field-aligned acceleration energy due to the field-aligned potential difference, $\Delta \Phi$. By replacing Eq. (2) in Eq. (1) one obtains:

$$
\begin{aligned}
j_{\|}\left(\Phi_{i}, \Phi_{m}\right)= & \Sigma_{P 0} \frac{d^{2} \Phi_{i}}{d x_{i}{ }^{2}}+\frac{a}{2 \sqrt{\epsilon_{\mathrm{em}}\left(\Phi_{i}, \Phi_{m}\right)}}\left(\frac{d \Phi_{i}}{d x_{i}}\right) \times \\
& \frac{d \epsilon_{\mathrm{em}}\left(\Phi_{i}, \Phi_{m}\right)}{d x_{i}}+a \sqrt{\epsilon_{\mathrm{em}}\left(\Phi_{i}, \Phi_{m}\right)} \frac{d^{2} \Phi_{i}}{d x_{i}{ }^{2}}
\end{aligned}
$$

The second and third term in the right-hand side of Eq. (4) describe the feedback; they vanish when $\Sigma_{P}$ is uniform and equal to the constant $\Sigma_{P 0}$.

The type of feedback described by Eqs. (2-4) has been considered in slightly different forms in previous models of quasi-static coupling between the magnetosphere and the auroral ionosphere. Lyons (1980) used the same formulas of Harel et al. (1977). In the model of Chiu and Cornwall (1980) the number of secondary electrons is taken proportional to $j_{\|}$. In these previous models the profile of the magnetospheric/generator potential, $\Phi_{m}$ is prescribed. Chiu and Cornwall (1980) obtained auroral arcs with spatial scales of the order of $40 \mathrm{~km}$ at $2000 \mathrm{~km}$ altitude. The novelty of our model with respect to previous kinetic ones is the selfconsistent description of the generator by a TD model and its coupling to the ionosphere, including the feedback described by Eq. (4).

The model is quasi-stationary. It describes processes with time scale $\left(\lambda_{T}\right)$ larger than: (1) $\tau_{e}$, the time needed for an electron to move from the magnetospheric generator to the ionospheric end of the magnetic flux tube and (2) $\tau_{A}$, the time needed for an Alfvén wave to travel between the two ends of the flux tubes. The time scale $\tau_{e}$ is related to the stationarity of the generator; it gives a measure of the time needed to empty/replenish the loss cone; $\tau_{A}$ is related to the 
time needed for a perturbation to propagate between the generator and the load and is a measure of the stationarity of the entire auroral circuit. Note that a $1 \mathrm{keV}$ electron travels along 20 Earth radii in about $7 \mathrm{~s}$ while the travel time of an Alfvén wave would be of the order of several minutes. In the next section we discuss numerical solutions of Eq. (4) obtained for various profiles of the generator potential, $\Phi_{m}$, corresponding to different values of plasma velocity, density and electron pressure at the inner edge of the LLBL.

\section{Quasi-stationary M-I coupling: numerical results}

\subsection{TD solution for the generator}

At the interface between the LLBL and the magnetospheric dusk flank we consider a right-handed reference frame with the interface layer containing the $(y-z)$ plane. As shown in Fig. 1a, the z-axis is aligned with the magnetic field direction, the $y$-axis is oriented anti-sunward along the LLBL velocity, and the x-axis ( $x_{m}$ in Fig. 1a) is perpendicular to the interface layer.

The transition between two asymptotic states of plasma and field variables in the generator is computed selfconsistently from the TD model of Roth et al. (1996). The boundary conditions of the TD model (bulk velocity, density, temperature) are specified at left in $x_{m \mathrm{~L}}$ (the MSPH side) and at right in $x_{m \mathrm{R}}$ (the LLBL side); $x_{m \mathrm{~L}}=-10000 \mathrm{~km}$ and $x_{m \mathrm{R}}=+5000 \mathrm{~km}$ in the examples discussed below. At the right hand-side of the TD the LLBL plasma bulk velocity, density and electron temperature take each of them a set of different values; the resulting TD solutions are plotted in Fig. 2. One color is assigned to solutions obtained for variations of one parameter $\left(V_{\mathrm{LLBL}}, n_{\mathrm{LLBL}}\right.$ or $\left.T_{\mathrm{LLBL}}^{-}\right)$; when a parameter is varied, the others take the reference value: $V_{\mathrm{LLBL}}=200 \mathrm{~km} / \mathrm{s}, n_{\mathrm{LLBL}}=5 \mathrm{~cm}^{-3}, T_{\mathrm{LLBL}}^{-}=10 \mathrm{eV}$. The parameters at the left hand-side of the TD, labeled MSPH, are kept constant for all the solutions illustrated in Fig. 2; their values are specified in Table 1 . Note that the overall magnetospheric transition layer maps into an ionospheric region extending roughly over $480 \mathrm{~km}$ in the $x_{i}$ direction. Figure 2 shows however only the central region $\left(-200 \mathrm{~km}<x_{m}<200 \mathrm{~km}\right)$ of the magnetospheric TD solution; the corresponding ionospheric projection extends over roughly $15 \mathrm{~km}$.

The various magnetospheric TD solutions illustrated in Fig. 2 show that the most significant changes of the magnetospheric potential, $\Phi_{m}$, are produced by variation of the LLBL bulk velocity, which is connected to the solar wind speed. A detailed view on the central region of the magnetospheric potential is given in Fig. 3 and illustrate small variations of $\Phi_{m}$, of the order of 100-200 V, introduced by variations of $n_{\mathrm{LLBL}}^{-}$and $T_{\mathrm{LLBL}}^{-}$at the right hand-side of the TD. The substructure of the magnetospheric potential evidenced in Fig. 3 maps into a thin ionospheric region with a thickness of about $2 \mathrm{~km}$. Figure 4 gives a detail of the plasma density profile and illustrates the effects on the density of changing the asymptotic parameters, $V_{\mathrm{LLBL}}, n_{\mathrm{LLBL}}^{-}$and $T_{\mathrm{LLBL}}^{-}$. The profiles of the magnetospheric potential, $\Phi_{m}$, as well as of the plasma density, $n$, illustrated in Figs. 2-4 are introduced in Eqs. (3) and (4) and the current continuity is then solved for the ionospheric potential, $\Phi_{i}\left(x_{i}\right)$.

\subsection{Solutions of the current continuity equation in the ionosphere}

We assume that the plasma and field properties vary only in the direction perpendicular to the arc. The current continuity equation is height-integrated up to the top of the ionosphere, at $z=z_{i}$, thus the problem is one-dimensional, with the spatial variable $x_{i}$ - the ionospheric coordinate normal to the arc at the ionospheric altitude (see Fig. 1b). The magnetospheric potential, $\Phi_{m}$, is given by the TD solution as a function of $x_{m}$ - the x-coordinate at magnetospheric altitude $z_{m}$. The profile of $\Phi_{m}$ has to be mapped into $\Phi_{m}\left(x_{i}\right)$ at the ionospheric altitude $z_{i}$, the mapping rule being determined by the topology of the magnetic field. In ERDK07 as well as in this paper we follow Lyons (1980) and use a simpler conical mapping with $x_{m}=x_{i} \sqrt{B_{i} / B_{m}}$. We consider a reference ionospheric altitude of $z_{i}=200 \mathrm{~km}$ and a magnetic compression factor $\sqrt{b}=32$.

The ionospheric feedback, $\Sigma_{P}=\Sigma_{P}\left(\epsilon_{\mathrm{em}}\right)$ included in Eq. (4) adds some nonlinearity to the current continuity equation solved as a two-point boundary problem. We noticed that the solution converges very slowly, if ever, when the boundary conditions, in $x_{i}=x_{i \mathrm{~L}}$ and $x_{i}=x_{i \mathrm{R}}$ correspond to a zero parallel potential drop (as in the work of Lyons, 1980), or a zero total field-aligned current density (as in ERDK07). The solution converges for a broader range of input parameters and the convergence is faster when the boundary conditions specify a vanishing flux of the precipitating energy, $\epsilon_{\mathrm{em}}=0$. The fluctuations of $\Sigma_{P}$ are therefore smoother close to the boundaries. This type of boundary condition can also be sustained on physical grounds by recalling that auroral arcs are structures localized spatially, defined by a significant electron precipitation that vanishes at the edges of the arc. We tested a range of values for $x_{i}=x_{i \mathrm{~L}}$ and $x_{i}=x_{i \mathrm{R}}$ for which the boundary condition, $\epsilon_{\mathrm{em}},=0$ was imposed. We chose limits large enough so that the overall profile of solutions of Eq. (4) did not vary anymore with $x_{i \mathrm{~L}}$ and $x_{i \mathrm{R}}$. In this way we are able to determine a self-consistent spatial scale of the discrete arc, independent of the boundary conditions. Self-consistent scales of the inverted-V region (tens to hundreds of kilometers) were obtained with quasi-static models by Chiu and Cornwall (1980). The partial current densities corresponding to all species treated in our model have been computed from the analytical expressions given in the Appendix of ERDK07. The numerical method used to solve Eq. (4) is based on finite differences and a damped Newton iterative procedure. The solutions are computed in 


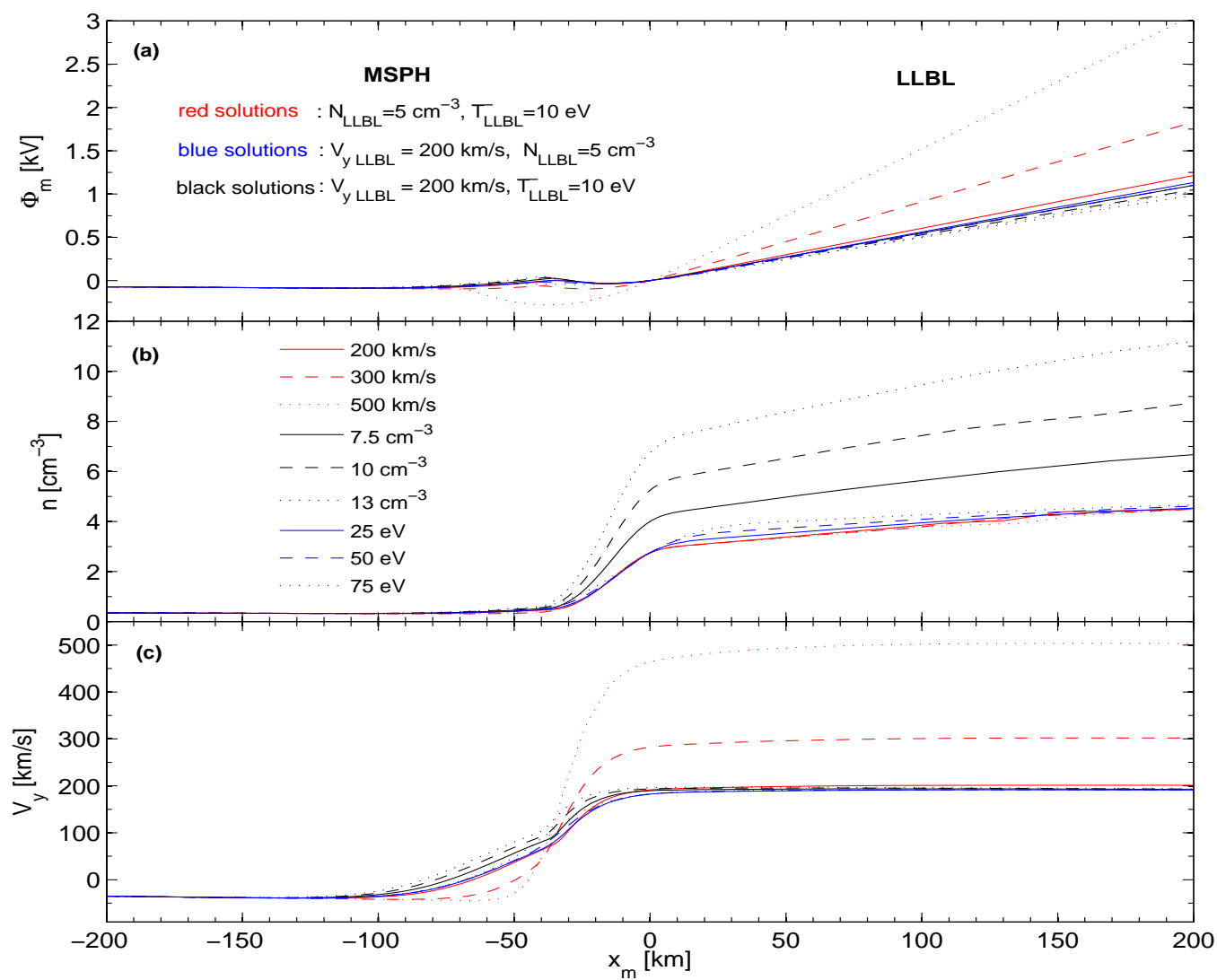

Fig. 2. Vlasov equilibrium solutions describing the magnetospheric generator. The panels show: (a) the magnetospheric potential, $\Phi_{m}$; (b) the plasma density, $n$; (c) plasma bulk velocity in the antinsunward direction, $V_{\mathrm{y}}$ (see also Fig. 1a-b). The magnetic field in $x_{m} \mathrm{~L}$, at the left (MSPH) side of the TD, is equal to $B_{z m}=30 \mathrm{nT}$. Each color corresponds to solutions obtained by changing one of the following asymptotic parameters at the right hand-side: LLBL bulk velocity (red), LLBL density (black), LLBL electron temperature (blue). Different linestyles illustrate solutions obtained for different values of the same asymptotic parameter, see legend of panel (b). When one parameter is varied the others take constant values: $V_{\mathrm{LLBL}}=200 \mathrm{~km} / \mathrm{s}, n_{\mathrm{LLBL}}=5 \mathrm{~cm}^{-3}, T_{\mathrm{LLBL}}^{-}=10 \mathrm{eV}$ (see also text inserted in panel a). The asymptotic parameters at the left hand-side (MSPH) are constant and given in Table 1 . The integration is carried out between $x_{m} \mathrm{~L}=-10000 \mathrm{~km}$ and $x_{m \mathrm{R}}=5000 \mathrm{~km}$ but only the central part of the solutions $\left(-200 \mathrm{~km}<x_{m}<+200 \mathrm{~km}\right)$ is shown in order to better visualize differences between the different profiles.

2000 spatial samples corresponding to a resolution of $50 \mathrm{~m}$ at $200 \mathrm{~km}$ altitude.

The solutions of Eq. (4), with the boundary conditions outlined above, might give a negative potential drop, $\Delta \Phi<0$ in regions adjacent to the upward field-aligned current. A negative $\Delta \Phi$ applied directly to the topside ionosphere will evacuate an important fraction of the ionospheric electrons, thus driving an unrealistically huge downward (or "return") current. Negative field-aligned potential drops must therefore be localized to higher altitudes, and the electrostatic potential might not vary monotonically with the altitude as was pointed out by Temerin and Carlson (1998). Therefore the current-voltage relationship derived by Knight (1973) is not valid in regions with negative field-aligned potential drop. Note that current-voltage relations for downward currents with non-monotonic variation of $\Delta \Phi$ with the altitude have been recently computed by Cran-McGreehin and
Wright (2005) and Vedin and Rönnmark (2005) but these relationships are not included in our model.

We assume as Temerin and Carlson (1998) that at ionospheric altitudes a negative field-aligned potential drop is "screened" by the ambipolar electric field. Thus, due to uncertainties in the current-voltage relationship we assume that when $\Delta \Phi<0$ the downward field-aligned current density of up-going ionospheric electrons is equal to the current density of up-going ionospheric ions. This limitation in the treatment of downward current reflects our partial lack of knowledge regarding the current-voltage relation explained above. Note also that in-situ measurements by Freja satellite (Marklund et al., 1997) suggest that the downward currents are associated to black aurora and are carried mainly by ionospheric electrons in thin regions with intense electric fields detected at altitudes higher than $200 \mathrm{~km}$. Owing to the non-linear current-voltage relation, negative current densities 


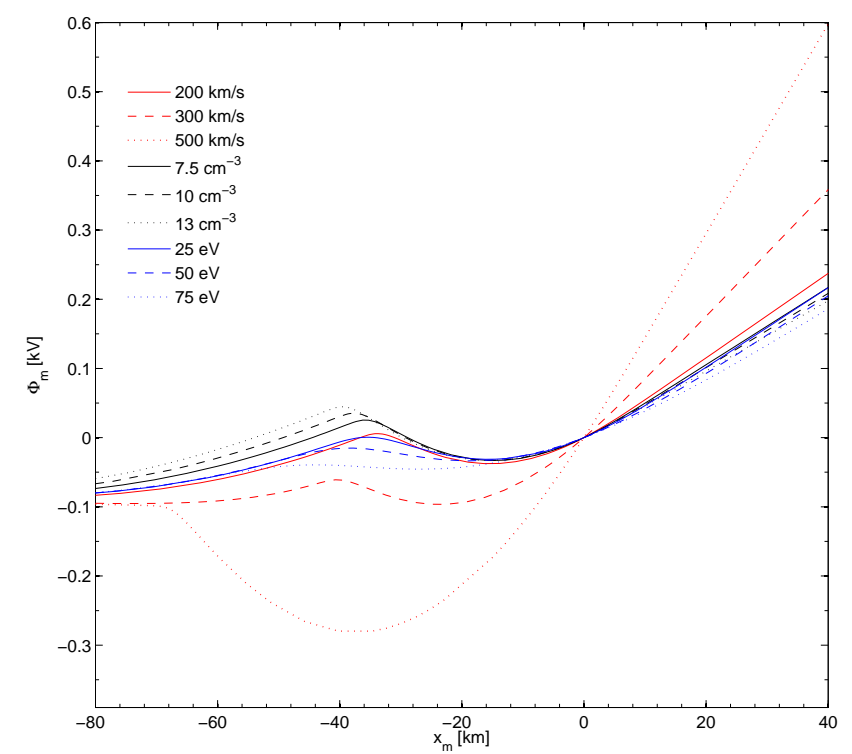

Fig. 3. Detail on the very central region of the magnetospheric potential profile, $\Phi_{m}\left(x_{m}\right)$, derived for TD solutions obtained for various boundary conditions: three LLBL bulk velocities (red profiles), three LLBL densities (black profiles), three LLBL electron temperatures (blue profiles). See caption of Fig. 2 for explanation of colors and reference values of parameters.

(corresponding to downward currents carried by ionospheric electrons) are obtained with our model for very small positive values of $\Delta \Phi$, as illustrated by some examples in the next sections.

\subsubsection{Effects due to variation of the LLBL velocity}

Figure 5 shows the solution of the current continuity Eq. (4) resulting for various profiles of $\Phi_{m}$ derived for different LLBL velocities (illustrated by red curves in Fig. 2). In Fig. 5 the Pedersen conductance is given by Eq. (2) with $\Sigma_{P 0}=0.5 \mathrm{~S}$, corresponding to a nightside background conductance. The structures obtained at $200 \mathrm{~km}$ altitude are quite narrow. We show the overall solution in panel (a) while panels $(b-d)$ zoom on the central region. The field-aligned potential drop, $\Delta \Phi$, increases with increasing LLBL bulk velocity. A similar result has been obtained with a uniform Pedersen conductance (ERDK07). The maximum value of $\Delta \Phi$ varies from $250 \mathrm{~V}$ to $2000 \mathrm{~V}$ when the LLBL velocity varies from $100 \mathrm{~km} / \mathrm{s}$ to $500 \mathrm{~km} / \mathrm{s}$ and the other plasma parameters are kept constant and equal to the reference values given in Tables 1 and 2. The relative peak observed in the profile of $\Delta \Phi$ (Fig. 3a) corresponds to the fine structure of $\Phi_{m}$ (see Fig. 2a).

The field-aligned current density, the energy flux of precipitating electrons, and the Pedersen conductance all increase with increasing LLBL speed. It is appropriate to consider that the luminosity of an auroral arc is proportional to

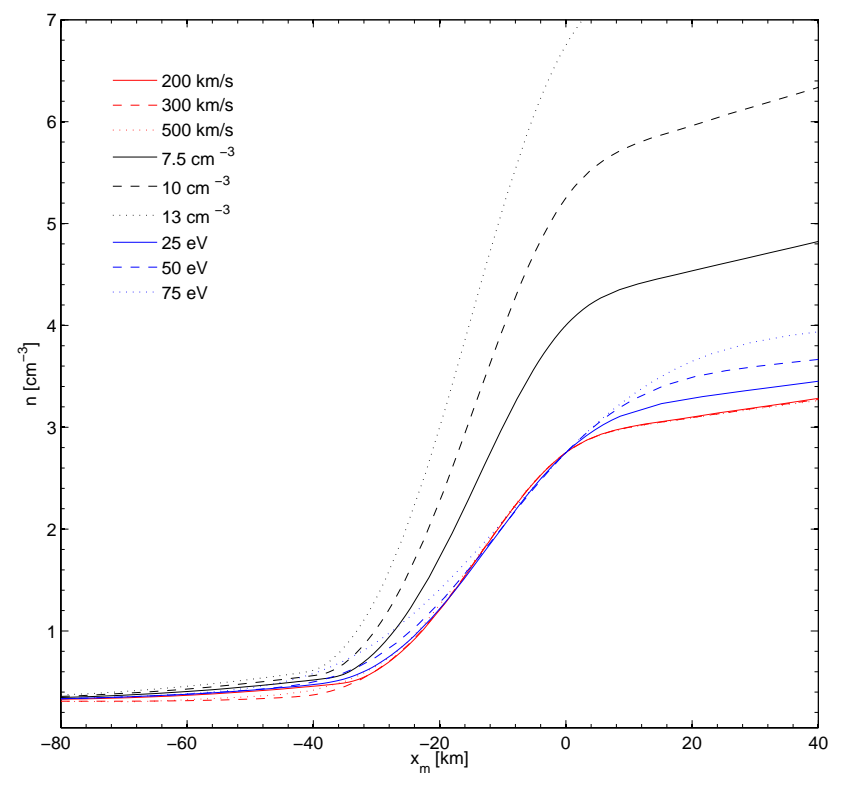

Fig. 4. Detail on the very central region of the magnetospheric electron density profile, $n\left(x_{m}\right)$, derived for TD solutions obtained for various boundary conditions: three LLBL bulk velocities (red profiles), three LLBL densities (black profiles), three LLBL electron temperatures (blue profiles). See also caption of Fig. 2.

Table 1. Asymptotic values of the number density, temperature and bulk velocity of the asymptotic populations that define the TD transition. "-" and "+" stand for electron and, respectively, proton. $l_{M, L}^{ \pm}$is a parameter of the VDF that controls the width of the TD layer (Echim et al., 2007).

\begin{tabular}{ccccccc}
\hline & $\begin{array}{c}n^{-}=n^{+} \\
{\left[\mathrm{cm}^{-3}\right]}\end{array}$ & $\begin{array}{c}T^{-} \\
{[\mathrm{eV}]}\end{array}$ & $\begin{array}{c}T^{+} \\
{[\mathrm{eV}]}\end{array}$ & $\begin{array}{c}V \\
{[\mathrm{~km} / \mathrm{s}]}\end{array}$ & $l^{-}$ & $l^{+}$ \\
\hline $\begin{array}{c}x=-\infty \text { (MSPH) } \\
x=+\infty \text { (LLBL) }\end{array}$ & 0.5 & 200 & 1000 & 0 & 20 & 5 \\
\hline
\end{tabular}

the flux of precipitating energy, $\epsilon_{e m}$. Therefore one can infer information about the auroral arc by inspecting the spatial variation of $\epsilon_{e m}$. The flux of precipitating energy obtained for $V_{\mathrm{LLBL}}=100 \mathrm{~km} / \mathrm{s}$ is of the order of $0.001 \mathrm{~W} \mathrm{~m}^{-2}$, the threshold limit of arc visibility with the unaided eye. Thus a sheared LLBL flow with a bulk velocity less than $100 \mathrm{~km} / \mathrm{s}$ would not supply enough energy to produce visible auroral luminosity.

An interesting feature of the solution is the discrete auroral arc centered in $x_{i}=0$ and extending over a couple of kilometer. The arc is embedded into a broader structure with a lower flux of precipitating energy and field-aligned current density. The dimmer aurora extends over a distance of 50 $60 \mathrm{~km}$. Note also the asymmetry of the profiles of $\Delta \Phi, j_{\|}$ and $\epsilon_{\mathrm{em}}$ that reflects an asymmetry of $\Phi_{m}$ and plasma density 

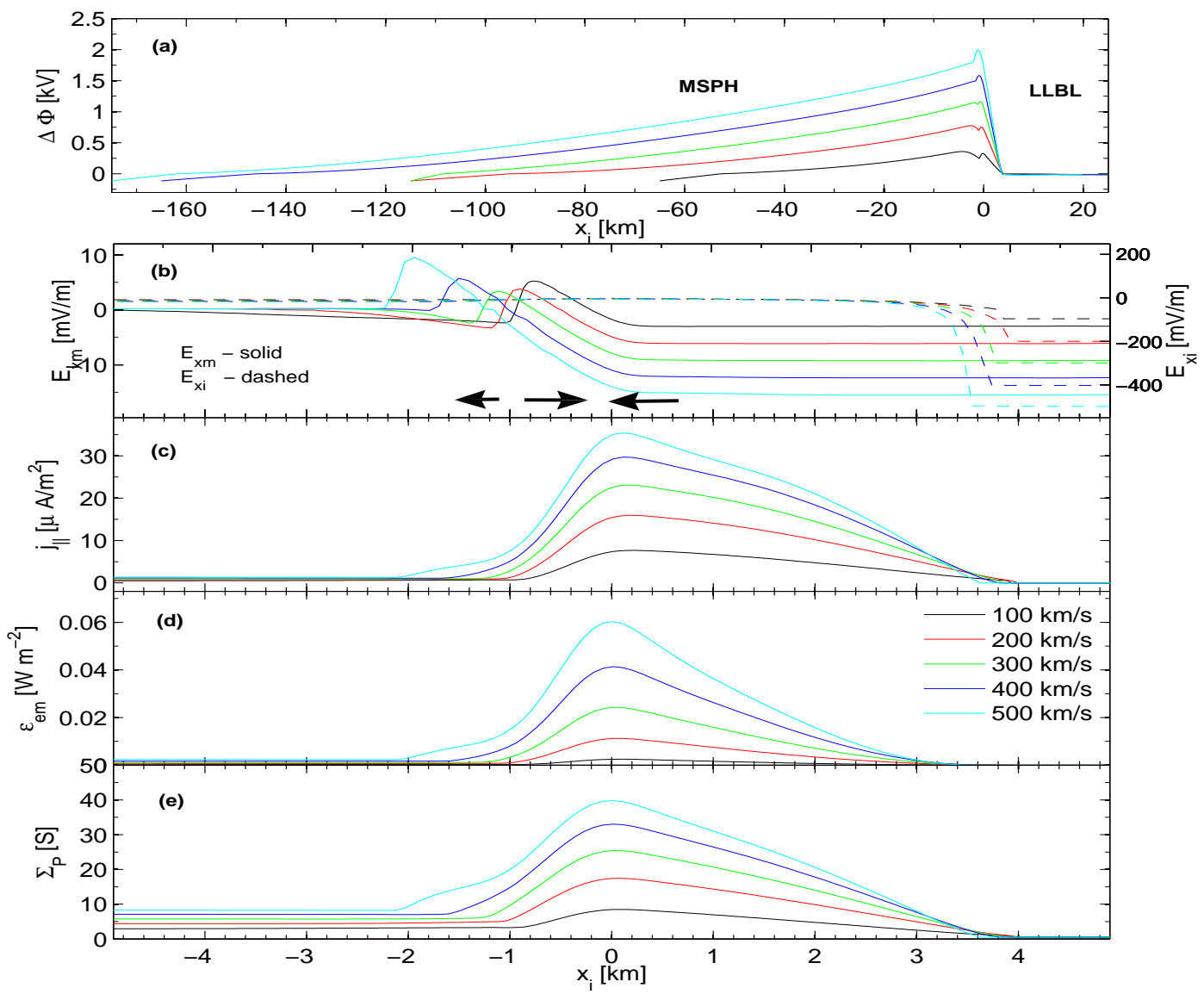

Fig. 5. Solution of Eq. (4) and the derived quantities obtained for $\Sigma_{P}$ given by Eq. (2) with $\Sigma_{P 0}=0.5$ and various profiles of $\Phi_{m}$ (see Fig. 2) obtained for five different bulk velocities on the LLBL side of the TD. The values of $V_{\text {LLBL }}$ corresponding to each color are given in the legend of panel (d). The panels show: (a) overall solution of the field-aligned potential drop; (b) detail of the profile of the magnetospheric (solid line) and ionospheric (dashed line) electric field; shown is the component normal to the arc; the solid arrows illustrate the orientation of the electric field suggesting the regions where the field is convergent or divergent; (c) detail of the profile of the field-aligned current density; (d) detail of the profile of the flux of precipitating energy; (e) detail of the profile of the height-integrated Pedersen conductivity.

Table 2. Reference density and temperature of the ionospheric species considered in the current-voltage relationship. The charge densities were adjusted such that charge neutrality is satisfied.

\begin{tabular}{cccc}
\hline & $\mathrm{e}^{-}$ & $\mathrm{O}^{+}$ & $\mathrm{H}^{+}$ \\
\hline $\mathrm{T}[\mathrm{eV}]$ & 0.2 & 0.02 & 0.02 \\
$\mathrm{n}\left[\mathrm{cm}^{-3}\right]$ & $7 \times 10^{3}$ & $6.5 \times 10^{3}$ & $5 \times 10^{2}$ \\
\hline
\end{tabular}

in the LLBL generator. The arc is localized close to the ionospheric mapping of the inner edge of the LLBL, northward of the region of lower (magnetospheric) precipitating energy flux. This feature of the solution is consistent with observations of discrete auroral arcs at the poleward side of the postnoon/evening auroral oval (Paschmann et al., 2003). The field-aligned current sheet (defined for $j_{\|}>1 \mu \mathrm{A} / \mathrm{m}^{2}$ ) is systematically broader than the discrete arc (corresponding to $\epsilon_{\mathrm{em}}>0.002 \mathrm{~W} \mathrm{~m}^{-2}$ ).
In Fig. 6 data from three classes of solutions are shown together: solutions obtained for $\Sigma_{P}$ given by Eq. (2) with $\Sigma_{P 0}=0.5 \mathrm{~S}$ (solid lines) and respectively $\Sigma_{P 0}=5.0 \mathrm{~S}$ (dashed lines); solutions obtained for a uniform $\Sigma_{P}$ equal to $\Sigma_{P 0}=5 \mathrm{~S}$ (dotted lines, discussed also in ERDK07). The ionospheric feedback results in: (1) an increase of the maximum field-aligned potential drops (see Fig. 6a); (2) an increase of the maximum energy flux of precipitating electrons (see Fig. 6b) and (3) an increase of the maximum fieldaligned current density (see Fig. 6c). The ionospheric feedback also produces narrower arcs (compare the squares on the dashed line with the squares on the dotted line in Fig. 6d) and field-aligned current channels (compare the circles on the dashed line with the circles on the dotted line in Fig. 6d). Thus the ionospheric feedback enhances the auroral characteristics observed for a uniform $\Sigma_{P}$. When the feedback is added to a background conductance of 5 Siemens the thickness of the field-aligned current sheet diminishes from $15 \mathrm{~km}$ to $10 \mathrm{~km}$. The width of the arc also decreases from 9 to $7 \mathrm{~km}$. 


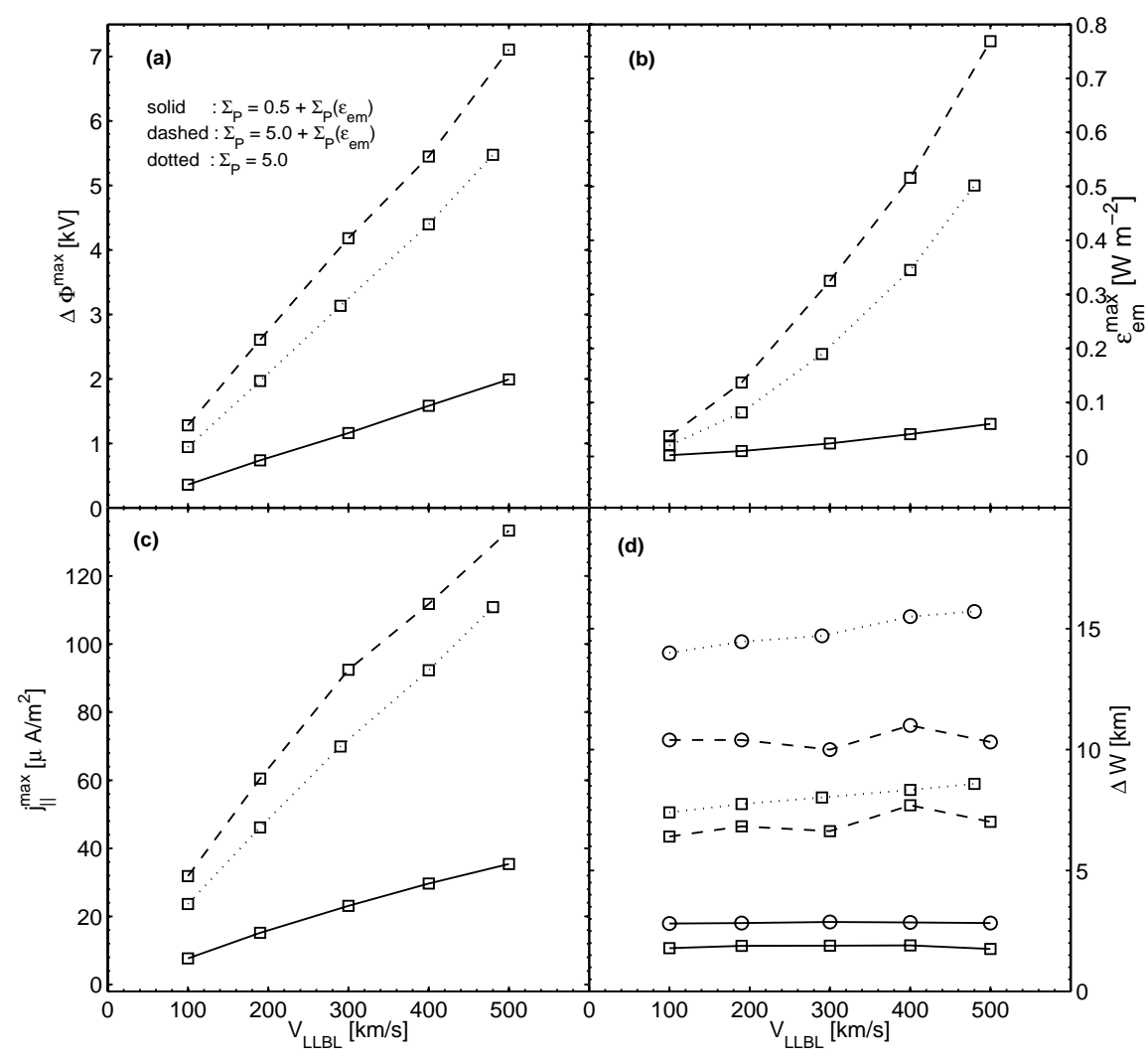

Fig. 6. Auroral arc's characteristics as a function of the bulk velocity at the LLBL side of the magnetospheric TD generator and heightintegrated Pedersen conductivity. Solid lines represents results obtained for a $\Sigma_{P}$ that depends on $\epsilon_{\mathrm{em}}$ (see Eq. 2) with a background conductance $\Sigma_{P 0}=0.5$; dashed lines show results obtained for a non-uniform conductance with $\Sigma_{P 0}=5$. $0 \mathrm{~S}$; dotted lines correspond to results obtained for a uniform Pedersen conductance, $\Sigma_{P}=5 \mathrm{~S}$. The panels show: (a) the variation with $V_{\text {LLBL }}$ of the maximum of the fieldaligned potential drop, $\Delta \Phi^{\max }$; (b) the variation with $V_{\mathrm{LLBL}}$ of the maximum of the flux of precipitating energy, $\epsilon_{e m}^{\mathrm{max}}$; (c) the variation with $V_{\text {LLBL }}$ of the maximum field-aligned current density, $j_{\|}^{\max }$; (d) the width at half maximum of the current sheet (circles) and of the auroral arc (squares) as a function of $V_{\mathrm{LLBL}}$.

The increase of $\Sigma_{P}$ by the flux of precipitating auroral electrons drives a reduction of the gradient of the electrostatic potential in the ionosphere. As the circulation of the electric field along the auroral circuit is equal to zero, a decrease of $d \Phi_{i} / d x_{i}$ produces an increase of the field-aligned potential drop, $\Delta \Phi$, precisely what is observed in the solutions outlined in Fig. 6. When the background conductance $\Sigma_{P 0}$ in Eq. (4) is reduced from $5 \mathrm{~S}$ to $0.5 \mathrm{~S}$, the values of the peak $\Delta \Phi$ are consistently smaller (compare the dashed and solid lines in Fig. 6a). A reduction of the fieldaligned current density and energy flux of precipitating electrons is also observed for smaller $\Sigma_{P 0}$. Changes in the background height-integrated conductivity, $\Sigma_{P 0}$, may reflect diurnal (dayside/nightside) or seasonal (summer/winter) variations. A low background Pedersen conductance favors less intense auroral arcs produced by electrons precipitating from the region close to the inner edge of the LLBL. Experimental studies at the dayside (Shue et al., 2001) seem to confirm this trend. The decreasing of $j_{\|}$with $\Sigma_{P 0}$ is mainly due to the reduction of the corresponding right-hand side term of the current continuity Eq. (4). The auroral structures are much narrower when $\Sigma_{P 0}$ diminishes from $5 \mathrm{~S}$ to $0.5 \mathrm{~S}$. The field-aligned current sheet extends roughly over $3 \mathrm{~km}$ while the thickness of the discrete arc is of the order of $2 \mathrm{~km}$.

Figure 6 shows that the maximum of the field-aligned potential drop, $\Delta \Phi^{\max }$, the maximum of the energy flux of precipitating electrons, $\epsilon_{\mathrm{em}}^{\max }$, and the maximum of the field-aligned current density, $j_{\|}^{\max }$, increase with increasing $V_{\text {LLBL }}$. This is a direct effect of the changes induced in the generator by an increased velocity shear. The TD interface generates more electromotive force when the LLBL and solar wind velocity is higher. While in the case of a uniform $\Sigma_{P}$ it seems that an increase of $V_{\text {LLBL }}$ produces slightly broader auroral structures, this effect is not seen when the ionospheric feedback is taken into account (see the square-dashed profile in Fig. 6d). Thus faster flows in the LLBL would produce brighter arcs without modifying their thickness. 


\subsubsection{Effects due to variation of the LLBL density}

The variation of the plasma density and its gradient in the magnetospheric generator has also effects on the coupling between the generator and the auroral ionosphere. These effects are illustrated in Fig. 7, showing the electrodynamic parameters obtained in the ionosphere for various magnetospheric $\Phi_{m}$ profiles derived for different values of $n_{\text {LLBL }}^{-}$, the LLBL electron density. Note that the density is increased only at the right-hand side of the TD generator, thus the pressure gradient across the TD is also increased. The results shown in Fig. 7 were obtained by solving Eq. (4) with an input magnetospheric potential, $\Phi_{m}$, illustrated by the black curves in Fig. 2a, corresponding to $V_{\mathrm{LLBL}}=200 \mathrm{~km} / \mathrm{s}$ (see Tables 1 and 2 for the values of the other plasma parameters). The height-integrated Pedersen conductivity is computed from Eq. (2) with $\Sigma_{P 0}=0.5 \mathrm{~S}$.

The field-aligned potential drop is smaller for increasing values of the LLBL density, as shown by Fig. 7a. On the contrary, the field-aligned current density increases with increasing $n_{\mathrm{LLBL}}^{-}$, an effect due primarily to the variation of $j_{\|}$with $n_{\text {LLBL }}^{-}$from the current-voltage relation (Knight, 1973). An interesting feature of this set of solutions is the negative spike of the field-aligned current density obtained at $x_{i}=2.75 \mathrm{~km}$ for the highest asymptotic LLBL density $\left(13 \mathrm{~cm}^{-3}\right)$ used in this study (see Fig. 7b). This peak of negative current, whose spatial scale is of the order of roughly $50 \mathrm{~m}$ at $200 \mathrm{~km}$ altitude, is obtained for small values of $\Delta \phi$, a consequence of the non linear current-voltage relation. Indeed when the condition $\Delta \Phi<k T_{i}^{-} / e$ is satisfied, with $T_{i}^{-}$the temperature of the ionospheric electrons, the flux of escaping ionospheric electrons is much larger than the flux of precipitating magnetospheric electrons (Knight, 1973). Since we consider an ionospheric population with $T_{i}^{-}=2320 \mathrm{~K}(\approx 0.2 \mathrm{eV})$, negative currents carried by ionospheric electrons are obtained only for a narrow range of values, $0<\Delta \Phi<0.2 \mathrm{~V}$, as illustrated by the negative peak in Fig. $7 \mathrm{~b}$.

Figure 8 summarizes the ionospheric response to changes of the LLBL density in the generator. It also shows how these effects depend on three different models of the heightintegrated Pedersen conductivity: uniform $\Sigma_{P}=5.0 \mathrm{~S}$ (dotted lines), non-uniform $\Sigma_{P}$ given by Eq. (2) with $\Sigma_{\mathrm{P} 0}=0.5 \mathrm{~S}$ (solid lines) and respectively with $\Sigma_{\mathrm{P} 0}=5.0 \mathrm{~S}$ (dashed lines). Regardless the model assumed for the conductivity, the peak of the field-aligned potential drop, $\Delta \Phi^{\max }$, decreases when LLBL density increases and seems to saturate for larger densities. This trend is consistent with Freja observations reported by Olsson and Janhunen (2000) for potential differences below $5 \mathrm{kV}$, in the 14:00-22:00 MLT sector. The maximum of the current density, $j_{\|}^{\max }$, increases with $n_{\mathrm{LLBL}}^{-}$and its variation is non-linear as shown by Fig. 8c.

The variation of $j_{\|}^{\max }$ and $\Delta \Phi^{\max }$ with the density in the generator does not follow exactly a linear approximation of the current-voltage relation (Lyons, 1980):

$j_{\|}=\frac{e^{2}}{\sqrt{2 \pi m^{-}}} \frac{n^{-}}{\sqrt{K T^{-}}} \Delta \Phi$

Note also that outside the discrete arc, for $-1 \mathrm{~km}<x_{i}<2 \mathrm{~km}$, both $j_{\|}$and $\Delta \Phi$ decrease with increasing $n_{\text {LLBL }}^{-}$. These effects illustrate the complex intermingling between parameters describing the electrodynamics at the ionospheric level $\left(j_{\|}\right)$, the parameters describing the generator state $\left(n_{\mathrm{LLBL}}^{-}\right)$and the potential drop between the generator and the load $(\Delta \Phi)$.

The magnetospheric potential, $\Phi_{m}$ is less affected by an increase of LLBL density from 5 to $13 \mathrm{~cm}^{-3}$ when the shear of velocity across the interface is $200 \mathrm{~km} / \mathrm{s}$. However the plasma density at the two sides of the TD generator is an important factor for the lifetime and sustainability of the overall TD structure. The solution of Eq. (4) shows strong variations with $n_{\text {LLBL }}^{-}$due to the role of this parameter in the currentvoltage relationship.

When the ionospheric feedback is included in the coupling model (solid and dashed lines in Fig. 8), the maximum of the energy flux of precipitating electrons, $\epsilon_{\mathrm{em}}^{\max }$, tends to decrease with increasing density of the magnetospheric generator. For a uniform $\Sigma_{P}$ (dotted lines in Fig. 8) the tendency is reversed and $\epsilon_{\mathrm{em}}$ increases with $n_{\mathrm{LLBL}}^{-}$. The auroral arc and field-aligned current sheet are narrower for an increased LLBL density in the generator. A drastic reduction of all auroral ionospheric parameters is observed when the background conductivity decreases from 5.0 Siemens to 0.5 Siemens, confirming that, for a broad range of generator parameters, a reduced background conductance diminishes the auroral effects in the postnoon/evening sector coupled to LLBL.

\subsubsection{Effects due to variation of the LLBL electron temper-} ature

An increase of the electron temperature, $T_{\mathrm{LLBL}}^{-}$, at the right hand-side of the magnetospheric generator results into an increase of the corresponding Larmor radius. The latter is the smallest spatial scale intervening in the magnetospheric TD solution. As can be seen also from Fig. 2 (solutions in blue) the effects on the amplitude of $\Phi_{m}$ due to increasing $T_{\mathrm{LLBL}}^{-}$ are quite limited. Nevertheless the small-scale peak revealed in Fig. 3 is significantly reduced for larger $T_{\mathrm{LLBL}}^{-}$. The temperature of the colder electron population at the inner edge of the LLBL is also an input parameter for the current-voltage relationship and the energy flux of precipitating electrons. Figures 9 and 10 summarize the effects of this parameter on the solution of the current continuity Eq. (4)

A remarkable feature of solutions shown in Fig. 9 is the region to the right of $x_{i}=5 \mathrm{~km}$, where the field-aligned potential drop takes negative values for LLBL electron temperatures higher than $10 \mathrm{eV}$. The largest negative values of 

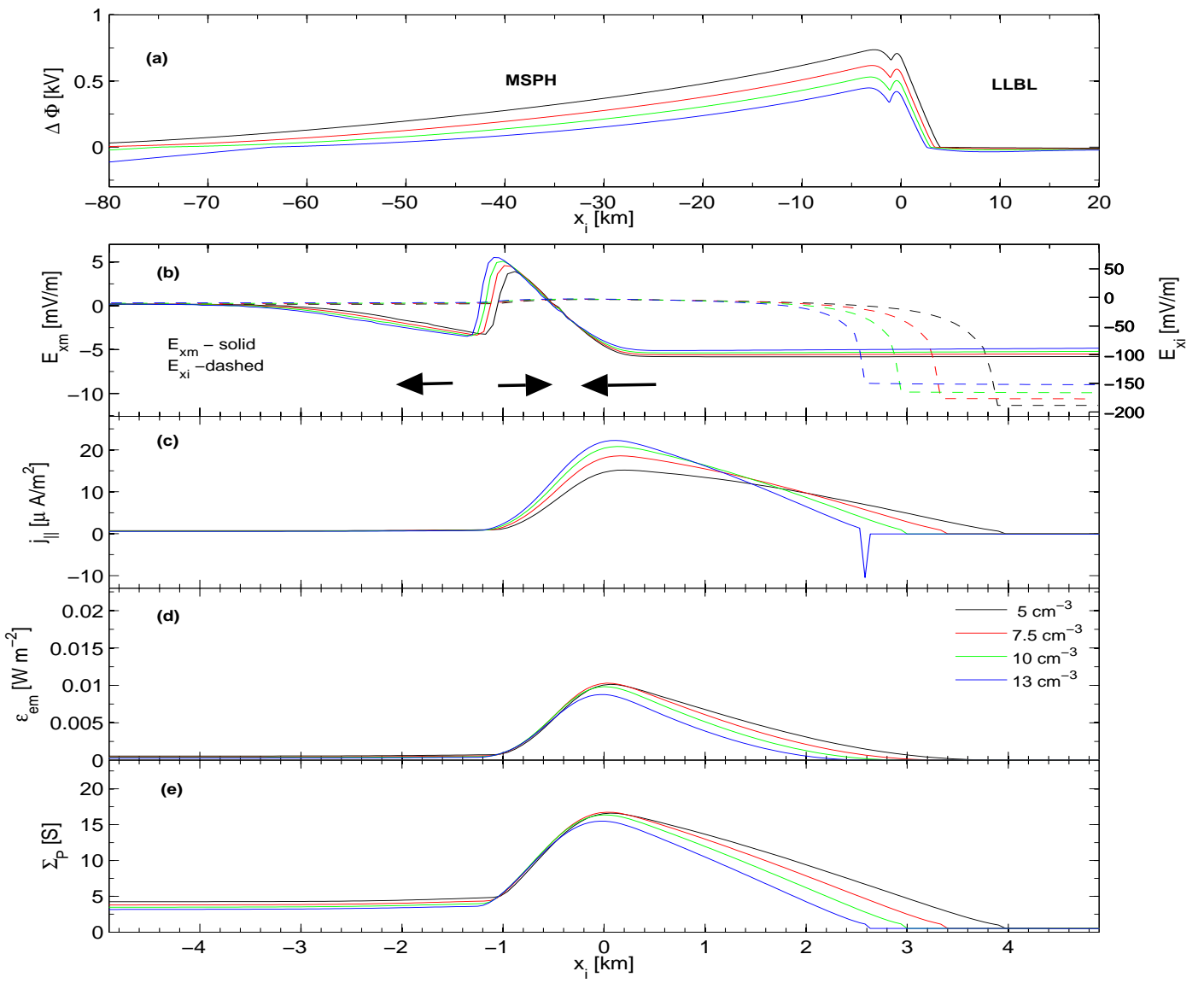

Fig. 7. Solutions of Eq. (4) and derived quantities obtained for a non-uniform height-integrated Pedersen with background conductivity $\Sigma_{P 0}=0.5$ (see Eq. 2). Different colors illustrate solutions obtained for various profiles of $\Phi_{m}$ obtained for four different LLBL plasma densities. The values of $n_{\text {LLBL }}^{-}$corresponding to each color are given in the legend of panel (d). See caption of Fig. 5 for an explanation of data from all panels.

$\Delta \Phi$ are obtained for the highest LLBL electron temperatures. In order to avoid unrealistically large field-aligned current densities in this region, the current density due to upgoing ionospheric electrons is set equal to the current density of upgoing ionospheric ions. This limitation is equivalent to imposing the condition that the ambipolar parallel electric field "screens" the negative field-aligned potential drop that is therefore confined at higher altitudes. The flux of precipitating magnetospheric protons gives a small current density, even for negative $\Delta \Phi$, suggesting that the downward current is mainly carried by ionospheric electrons as shown by Marklund et al. (1997). In Fig. 9a the amplitude of $\Delta \Phi$ increases with $T_{\mathrm{LLBL}}^{-}$for each $x_{i}$. The field-aligned current density increases with $T_{\mathrm{LLBL}}^{-}$only outside a thin region defined by $-0.75 \mathrm{~km}<x_{i}<3 \mathrm{~km}$; inside this region $j_{||}$decreases with $T_{\text {LLBL }}^{-}$.

Figure 10 summarizes the effects of increasing LLBL electron temperature on the coupling between the magnetospheric TD generator and the auroral ionosphere. A saturation effect is obtained for $j_{\|}^{\max }$ and $\Delta \Phi^{\max }$; the max- imum of current density decreases while the maximum of field-aligned potential drop increases with increasing $T_{\mathrm{LLBL}}^{-}$. This behavior is observed for all three models adopted for the height-integrated Pedersen conductivity. The maximum of the energy flux of precipitating electrons decreases with increasing $T_{\mathrm{LLBL}}^{-}$for $\Sigma_{P}$ given by Eq. (2) and $\Sigma_{\mathrm{P} 0}=5$ (dashed lines in Fig. 10c). This effect is less evident for the other two models adopted for $\Sigma_{P}$. The auroral structures have the tendency to expand spatially for larger electron temperature in the generator for all three models of $\Sigma_{P}$. This expansion is due to the increase of the smallest spatial scale of the generator. In summary, Fig. 10 shows that auroral arcs are brighter and thinner for a colder electron population in the solar wind and LLBL. As in previous cases, for all the temperatures investigated, the auroral ionospheric parameters take significantly larger values when the background conductivity is larger. 


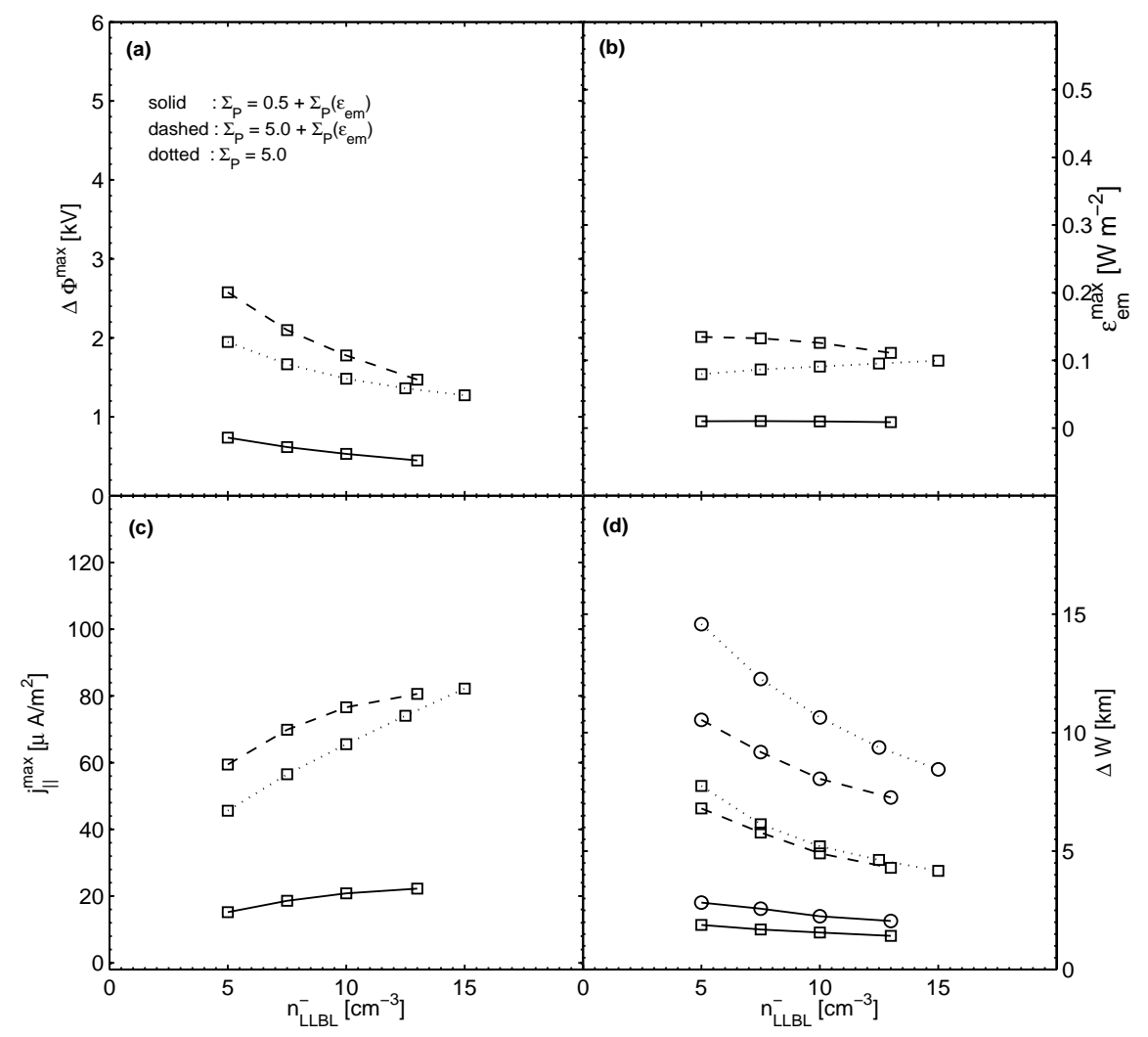

Fig. 8. Auroral arc's characteristics as a function of: (a) the LLBL plasma density and (b) the height-integrated Pedersen conductivity. Solid lines represents results obtained for $\Sigma_{P}$ depending on $\epsilon_{\text {em }}$ (see Eq. 2) with a background conductance $\Sigma_{P 0}=0.5$; dashed lines show results obtained for a non-uniform conductance with $\Sigma_{P 0}=5.0 \mathrm{~S}$; dotted lines correspond to results obtained for a uniform Pedersen conductance, $\Sigma_{P}=5 \mathrm{~S}$. Same scales are used as in Fig. 6 for a better comparison of the results. See also caption of Fig. 6 for a description of panel data.

\section{Summary and discussion}

The results discussed in this paper describe the coupling between a sheared flow layer at the inner edge of the LLBL and the polar ionosphere. The TD solution assumed for the magnetospheric sheared flow describes a generator of electromotive force with $\boldsymbol{\nabla} \cdot \boldsymbol{E}<0$. The generator "power" is mainly determined by the shear of velocity and/or the gradients of density and temperature driven by the interaction between the solar wind and the magnetosphere in the dusk flank. The profile of transition between these two asymptotic states is computed self-consistently from Vlasov and Maxwell's equations. The resulting magnetospheric electrostatic potential is introduced into the current continuity equation at the top of the ionosphere. The current continuity equation couples the magnetospheric and ionospheric plasma and field parameters. The field-aligned current density is a function of the field-aligned potential drop inferred from the current-voltage relationship derived from a kinetic treatment of the adiabatic motion of particles moving between both ends of the magnetic flux tube.

On one hand, an increase of LLBL and solar wind velocity, and implicitely of the velocity shear across the boundary layer, modifies the magnetospheric potential and increases the electric field at the inner edge of the LLBL. On the other hand, variations of the LLBL temperature and density, related to solar wind changes, do not produce significant variations of $\Phi_{m}$ but may have significant effects, via the currentvoltage relationship, on the field-aligned current density and consequently on the solution of the current continuity equation. This aspect is disregarded in models assuming a linear current-voltage relationship and uniform plasma density and temperature in the generator.

The model for the M-I coupling discussed in this paper shows a number of interesting effects on the auroral arc characteristics due to variations of plasma properties in the LLBL. The field-aligned current density and the flux of precipitating energy, and thus also arc luminosity, increase with increasing velocity shear at the inner edge of the LLBL. They reach values of the order of tens of $\mu \mathrm{A} / \mathrm{m}^{2}$ and, respectively, tens of $\mathrm{mW} / \mathrm{m}^{2}$ for a velocity shear in the LLBL of the order of $500 \mathrm{~km} / \mathrm{s}$ and a field-aligned potential drop of the order of $2 \mathrm{kV}$. The Pedersen conductance takes values up to $30 \mathrm{~S}$ for a background $\Sigma_{P 0}=0.5 \mathrm{~S}$. For the parameters tested in this study (see Tables 1 and 2) we found a threshold value of the velocity shear $(\approx 100 \mathrm{~km} / \mathrm{s})$ below which the flux of 

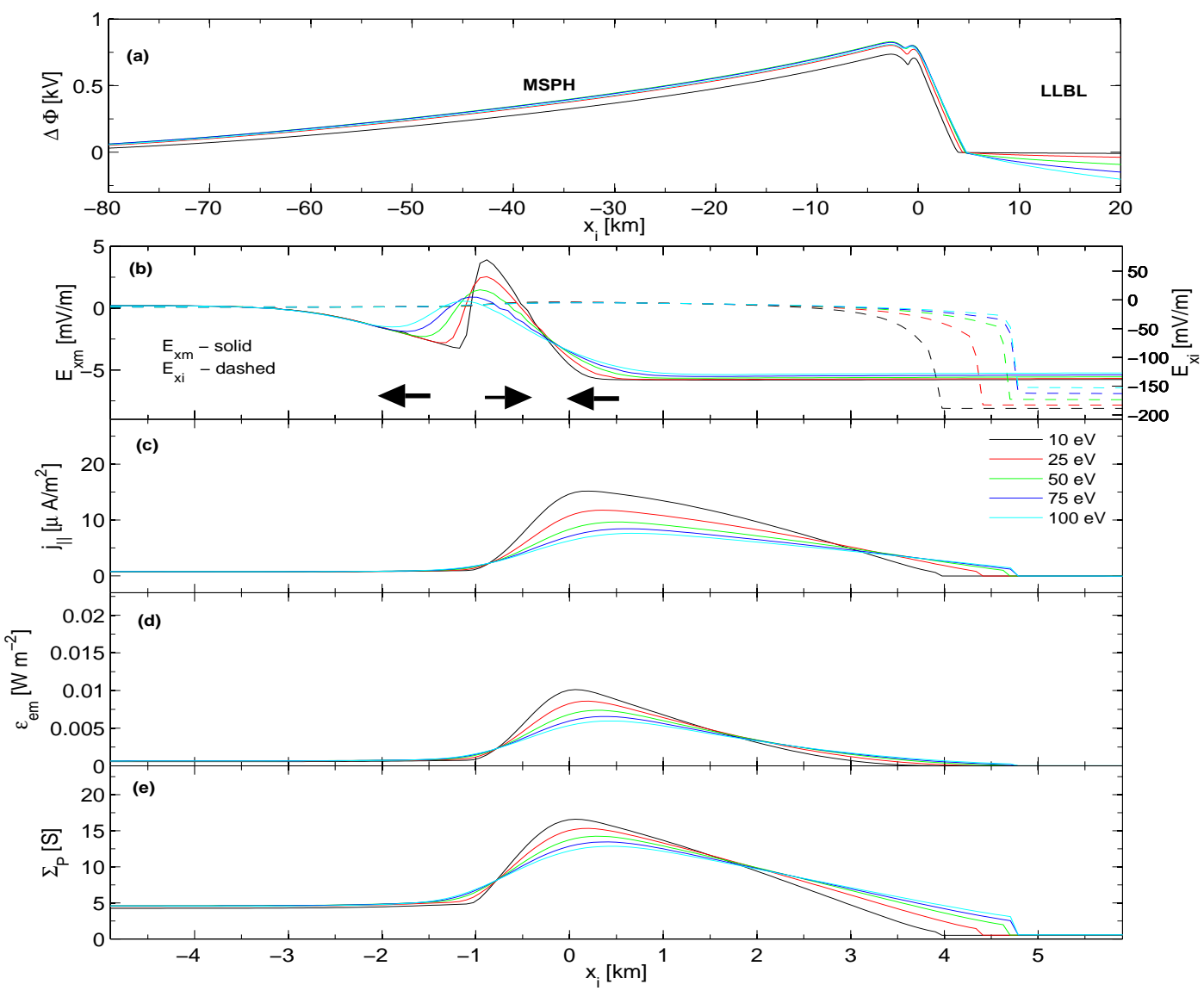

Fig. 9. Solutions of Eq. (4) and derived quantities obtained for a non-uniform height-integrated Pedersen conductivity with background $\Sigma_{P 0}=0.5$ (see Eq. 2). Different colors illustrate solutions obtained for various profiles of $\Phi_{m}$ obtained for five different various electron temperatures at the LLBL side of the TD. The values of $T_{\mathrm{LLBL}}^{-}$corresponding to each color are given in the legend of panel (d) See caption of Fig. 5 for details.

precipitating energy is less than the threshold of visibility by human eye. In such cases the TD generator does not provide enough power to sustain visible auroral arcs. Increased densities at the LLBL side of the generator produce a narrowing of the auroral arc. The model also shows that the currentvoltage relationship is nonlinear in narrow regions where the field-aligned current density is maximal. Assuming that the plasma properties in the solar wind and LLBL slowly vary in time, the effects discussed above could also describe the time evolution of the auroral characteristics as a function of the (slowly) time change of the generator.

For a moderate background Pedersen conductance $\left(\Sigma_{P 0}=5 \mathrm{~S}\right)$ the ionospheric feedback enhances the magnetosphere-ionosphere coupling; the auroral effects are more prominent than in the case of a uniform $\Sigma_{P}$. Indeed, inside the arc $\Sigma_{P}$ increases due to precipitation of electrons and a smaller perpendicular electric field is established in the ionosphere. Since the circulation of the electric field in the auroral circuit is equal to zero, the reduction of the perpendicular E-field is necessarily accompanied by an enhancement of the field-aligned potential drop (Roth et al., 1993). Our model shows also that for values of $\Sigma_{P 0}$ of the order of $0.5 \mathrm{~S}$, one obtains smaller field-aligned potential drops, reduced field-aligned current densities and fluxes of precipitating energy as well as narrower auroral structures. Statistical studies (Shue et al., 2001) show that dayside global auroral brightness increases slightly with background Pedersen conductance in the range $0.5 \mathrm{~S}$ to $5 \mathrm{~S}$. The solutions obtained with ionospheric feedback also show negative potential drops in the neighborhood of the arc, at the poleward side, especially for increased thermal energy of the generator plasma.

In ideal MHD and fluid models of magnetosphereionosphere coupling, small-scale field-aligned currents and discrete arcs are obtained as a consequence of instabilities driven by small-scale perturbations of the ionospheric conductivity and Alfvén waves (Sato, 1978; Lysak, 1986). In time-dependent fluid models the initial state and the initial perturbartion are defined rather arbitrarily and the generator is introduced via an ad-hoc conductivity model and an 


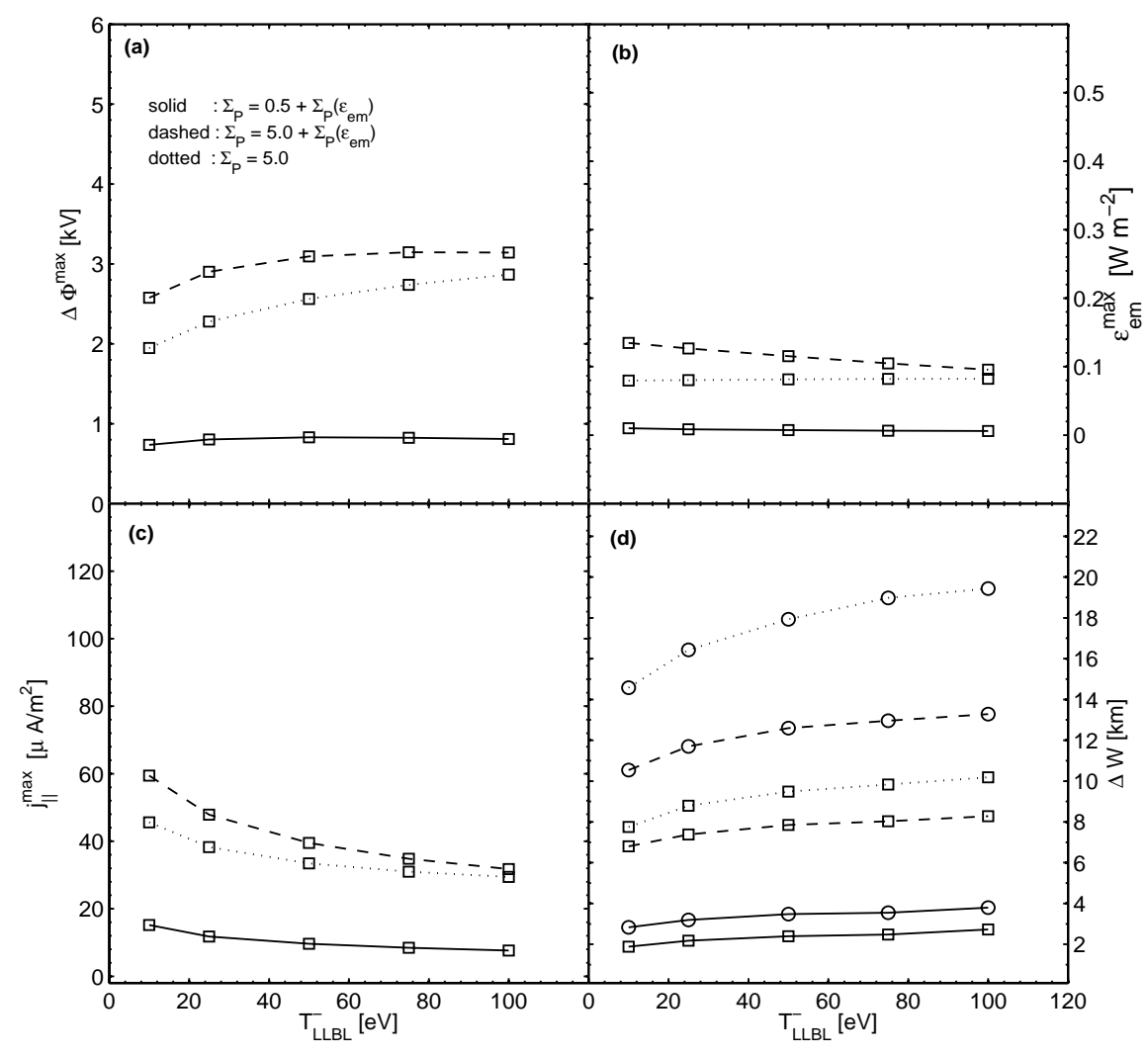

Fig. 10. Auroral arc's characteristics as a function of: (a) the LLBL electron temperature and (b) the height-integrated Pedersen conductivity. Solid lines represents results obtained for a $\Sigma_{P}$ that depends on $\epsilon_{\mathrm{em}}$ (see Eq. 2) with a background conductance $\Sigma_{P 0}=0.5$; dashed lines show results obtained for a non-uniform conductance with $\Sigma_{P 0}=5.0 \mathrm{~S}$; dotted lines correspond to results obtained for a uniform Pedersen conductance, $\Sigma_{P}=5 \mathrm{~S}$. Same scales are used as in Fig. 6 for a better comparison of the results. See also caption of Fig. 6 for a description of panel data.

inductance of the field lines connecting the generator and the load (Sato, 1978; Lysak, 1986). Models of the ionospheric feedback mechanism developped by Lysak (1986) and Lysak and Song (2002) (see also the references therein) give auroral arcs with scale length of the order of $10 \mathrm{~km}$; the upward field-aligned current density is of the order of $10 \mu \mathrm{A} / \mathrm{m}^{2}$ corresponding to Pedersen conductances of the order of 10-15 Siemens. In this paper we show that thinner arclike solutions can be obtained in the stationary case with a quasi-stationary model based on kinetic treatment of the adiabatic motion of charged particles and a Vlasov equilibrium solution for the magnetospheric generator with sheared plasma flow. We obtain also more intense field-aligned currents for roughly the same range of Pedersen conductances. In our model the spatial scale of the arc depends on the spatial scales of the generator; the latter are self-consistently determined and depend on the magnetospheric electron and proton Larmor radius.

Non-stationary aspects of the coupling between the LLBL generator and the auroral ionosphere were described by the fluid model of Lotko et al. (1987) based on a prescribed initial distribution of the electric field in the LLBL. When the enhancement of $\Sigma_{P}$ by auroral electrons is neglected, the non-steady/turbulent flow in the LLBL depends in Lotko et al.'s model on the Hartman number that in turn is determined by several parameters, including the background Pedersen conductance. Lotko et al. (1987) observed that the fieldaligned potential drop increases with the Hartman number a trend that could be considered similar to the variation of $\Delta \Phi$ with $\Sigma_{P}$ in our model. In Lotko et al. (1987) the spatial scale of the inner interface of the LLBL strongly depends on an ad-hoc viscosity coefficient; in our model the spatial length of the transition layer is determined self-consistently from asymptotic macroscopic plasma parameters and velocity distribution functions at the two sides of the transition region. Lotko et al. (1987) show also that a sheared flow in the LLBL of the order of $200 \mathrm{~km} / \mathrm{s}$ decays due to the viscous and electromagnetic coupling to the ionosphere in about 30-40 min. The TD generator considered in our model may be subject to a discharging due to Joule dissipation in the coupled ionosphere. Roth et al. (1993) have shown that the typical time for "discharging" a TD formed in a plasma with temperatures of the order of $1 \mathrm{keV}$ and densities of the order of $0.5 \mathrm{~cm}^{-3}$ is larger than $10 \mathrm{~min}$. 
The quasi-stationary model discussed in this paper describes the coupling between the LLBL and the polar ionosphere as a function of kinetic and macroscopic properties of the generator. The model has, however, some limitations. One simplification concerns the treatment of the downward field-aligned current. Whenever the field-aligned potential drop is negative, $\Delta \Phi<0$, the downward current is carried in our model only by precipitating magnetospheric ions. This limitation is imposed by the uncertainties of the currentvoltage relationship in the dowward current region. Significant downward currents, carried by ionospheric electrons, can be obtained, however, for minute positive values of $\Delta \Phi$ - a consequence of the non-linear current-voltage relationship (Knight, 1973).

Another simplification is the description of the magnetic field topology. We use a cylindrical geometry that simplifies the mathematics without obscuring the physics. In reality magnetic field lines that map in the LLBL are streched and their topology is more complicated. The geometry of the problem should not alter significantly the main results described in Sects. 3.2.1-3.2.3.

Time dependent effects are not considered in this study therefore the results cannot describe rapid variations observed in auroras. The model can be applied for auroral forms stable over intervals of several minutes, the time needed for an Alfvén wave to travel between the two ends of the auroral flux tube. Self-consistent feedback effects on the generator itself are also neglected. Indeed, we assume that the external driver, the solar wind in the case of the LLBL, supplies enough momentum and energy such that the generator maintains the "power" supplied to the coupled ionosphere.

The model discussed in this paper requires sheared plasma flows in the dusk LLBL. Possible physical mechanisms of sheared flows in the LLBL could be related to impulsively injected magnetosheath plasma elements and/or rolled-up Kelvin-Helmholtz vortices. Our results describe how the characteristics of auroral arcs in the post-noon and preevening sector change with changing solar wind and LLBL properties. Satellite and ground-based experimental data can offer deeper insight into the auroral plasma processes investigated by this study.

Acknowledgements. M. Echim is beneficiary of a fellowship granted by the Belgian Federal Science Policy Office (Project $\mathrm{MO} / 35 / 021$, "Electrodynamic coupling of the auroral ionosphere and magnetosphere (follow-up)" and acknowledges support from ESA (project PECS KEEV nr. 98049) and Romanian Agency for Science (project SAFIR nr. 81-009, Parteneriate-D8).

Topical Editor I. A. Daglis thanks J. Vogt and another anonymous referee for their help in evaluating this paper.

\section{References}

Atkinson, G.: Auroral arcs: result of the interaction of a dynamic magnetosphere with the ionosphere, J. Geophys. Res., 75, 47464755, 1970.

Bythrow, P., Heelis, R., Hanson, W., Power, R. A., and Hoffman, R. A.: Observational evidence for a boundary layer source of dayside region 1 field-aligned current, J. Geophys. Res., 86, 5577, 1981.

Chiu, Y. T. and Cornwall, J. M.: Electrostatic model of a quiet auroral arc, J. Geophys. Res., 85, 543-556, 1980.

Chiu, Y. T. and Schulz, M.: Self-consistent particle and parallel electrostatic field distributions in the magnetosphericionospheric auroral region, J. Geophys. Res., 83, 629-642, 1978.

Cran-McGreehin, A. and Wright, A.: Current-voltage relationship in downward field-aligned current region, J. Geophys. Res., 110, A10S10, doi:10.1029/2004JA010870, 2005.

De Keyser, J., Roth, M., and Lemaire, J.: The magnetospheric driver of subauroral ion drifts, Geophys. Res. Lett., 25, 16251628, doi:10.1029/98GL01135, 1998.

Drakou, E., Sonnerup, B., and Lotko, W.: Self-consistent steady state model of the low-altitude boundary layer, J. Geophys. Res., 99, 2351-2364, 1994.

Echim, M., Roth, M., and De Keyser, J.: Sheared magnetospheric plasma flows and discrete auroral arcs: a quasi-static coupling model, Ann. Geophys., 25, 317-330, 2007, http://www.ann-geophys.net/25/317/2007/.

Evans, D.: The characteristics of a persistent auroral arc at the high latitude in the 1400 MLT sector, in: The Polar Cusp, edited by: Holtet, J. and Egeland, A., pp. 99-109, D. Reidel, 1985.

Fridman, M. and Lemaire, J.: Relationship between auroral electron fluxes and field-aligned electric potential differences, J. Geophys. Res., 85, 664-670, 1980.

Hardy, D., Gussenhoven, M., and Brautigam, D.: A statistical model of auroral ion precipitation, J. Geophys. Res., 94, 370 392, 1989.

Harel, M., Wolf, R., Reiff, P., and Hillis, H.: Study of plasma flow near the Earth's plasmapause, Tech. Rep. AFGL-TR-77286, U.S. Air Force Geophys. Lab, 1977.

Hasegawa, A.: Particle acceleration by MHD surface wave and formation of aurora, J. Geophys. Res., 81, 5083-5090, 1976.

Iijima, T. and Potemra, T.: Amplitude distribution of field-aligned currents at northern high latitudes observed by Triad, J. Geophys. Res., 81, 2165-2174, 1976.

Knight, L.: Parallel electric fields, Planet. Space Sci., 21, 741-750, 1973.

Kozlovsky, A. and Kangas, J.: Characteristics of the postnoon auroras inferred from EISCAT radar measurements, J. Geophys. Res., 106, 1817-1834, 2001.

Lemaire, J.: Impulsive penetration of filamentary plasma elements into the magnetosphere of the Earth and Jupiter, Planet. Space Sci., 25, 887-890, 1977.

Lemaire, J. and Roth, M.: Penetration of solar wind plasma elements into the magnetosphere, J. Atmos. Terr. Phys., 40, 331$335,1978$.

Lemaire, J. and Scherer, M.: Plasma sheet particle precipitation: A kinetic model, Planet. Space Sci., 21, 281-289, 1973.

Lotko, W., Sonnerup, B. U. O., and Lysak, R. L.: Nonsteady boundary layer flow including ionospheric drag and parallel electric fields, J. Geophys. Res., 92, 8635-8648, 1987. 
Lummerzheim, D. and Lilensten, J.: Electron transport and energy degradation in the ionosphere: evaluation of the numerical solution, comparison with laboratory experiments and auroral observations, Ann. Geophys., 12, 1039-1051, 1994, http://www.ann-geophys.net/12/1039/1994/.

Lundin, R. and Evans, D.: Boundary layer plasmas as a source for high-latitude, early afternoon, auroral arcs, Planet. Space Sci., 33, 1389-1406, 1985.

Lundin, R. and Sandahl, I.: Some characteristics of the parallel electric field acceleration of electrons over discrete auroral arcs as observed from two rocket flights, Tech. Rep. SP-135, European Space Agency, 1978.

Lundin, R., Yamauchi, M., Woch, J., and Marklund, G.: Boundary layer polarization and voltage in the 14 MLT region, J. Geophys. Res., 100, 7587-7597, 1995.

Lyons, L.: Generation of large-scale regions of auroral currents, electric potentials and precipitation by the divergence of the convection electric field, J. Geophys. Res., 85, 17-24, 1980.

Lyons, L.: Discrete aurora as the direct result of an inferred high altitude generating potential distribution, J. Geophys. Res., 86, $1-8,1981$

Lyons, L., Evans, D., and Lundin, R.: An observed relation between magnetic field aligned electric fields and downward electron energy fluxes in the vicinity of auroral forms, J. Geophys. Res., 84, 457-461, 1979.

Lysak, R.: Coupling of the dynamic ionosphere to auroral flux tubes, J. Geophys. Res., 91, 7047-7056, 1986.

Lysak, R. and Song, Y.: Energetics of the ionospheric feedback interaction, J. Geophys. Res., 107, 1160, doi:10.1029/2001JA000308, 2002.

Marklund, G., Karlsson, T., and Clemmons, J.: On low-altitude particle acceleration and intense electric fields and their relationship to black aurora, J. Geophys. Res., 102, 17 509-17 522, 1997.

Marklund, G., Ivchenko, N., Karlsson, T., Fazakerley, A., Dunlop, M., Lindqvist, P.-A., Buchert, S., Owen, C., Taylor, M., Vaivads, A., Carter, P., André, M., and Balogh, A.: Temporal evolution of the electric field accelerating electrons away from the auroral ionosphere, Nature, 414, 724-727, 2001.

Miura, A. and Pritchett, P.: Nonlocal stability analysis of the MHD Kelvin-Helmholtz instability in a compressible plasma, J. Geophys. Res., 87, 7431-7444, 1982.

Moen, J., Sandholt, P., Lockwood, M., Egeland, A., and Fukui, K.: Multiple, discrete arcs on sunward convecting field lines in the 14-15 MLT region, J. Geophys. Res., 99, 6113-6123, 1994.

Newell, P., Ruohoniemi, J., and Meng, C.-I.: Maps of precipitation by source region, binned by IMF, with inertial convection streamlines, J. Geophys. Res., 109, A10206, doi:10.1029/2004JA010499, 2004.

Newell, P., Wing, S., and Meng, C.-I.: Spectral properties and source regions of dayside electron acceleration events, J. Geophys. Res., 110, A11205, doi:10.1029/2005JA011264, 2005.

Olsson, A. and Janhunen, P.: Difference in the current-voltage relationships between dawn and duskside inverted-V events, J. Geophys. Res., 105, 5373-5380, doi:10.1029/1999JA900504, 2000.

Paschmann, G., Haaland, S., and Treumann, R.: Auroral plasma physics, Kluwer Academic Publishers, first edn., 2003.

Pierrard, V.: New model of magnetospheric current-voltage relationship, J. Geophys. Res., 101, 2669-2676, 1996.

Pierrard, V., Khazanov, G. V., and Lemaire, J. F.: Current voltage relationship, J. Atmos. Sol.-Terr. Phy., 69, 2048-2057, doi:10. 1016/j.jastp.2007.08.005, 2007.

Robinson, R., Vondrak, R., Miller, K., Dabbs, T., and Hardy, D.: On calculating ionospheric conductances from the flux and energy of precipitating electrons, J. Geophys. Res., 92, 2565-2569, 1987.

Rönnmark, K. and Hamrin, M.: Auroral electron acceleration by Alfvn waves and electrostatic field, J. Geophys. Res., 105, $25333-25344,2000$

Roth, M., Evans, D., and Lemaire, J.: Theoretical structure of a magnetospheric plasma boundary: application to the formation of discrete auroral arcs, J. Geophys. Res., 98, 11411-11423, 1993.

Roth, M., de Keyser, J., and Kuznetsova, M.: Vlasov Theory of the Equilibrium Structure of Tangential Discontinuities in Space Plasmas, Space Sci. Rev., 76, 251-317, 1996.

Sato, T.: A theory of quiet auroral arcs, J. Geophys. Res., 83, 1042 $1048,1978$.

Sergienko, T. and Ivanov, V.: A new approach to calculate the excitation of atmospheric gases by auroral electron impact, Ann. Geophys., 11, 717-727, 1993, http://www.ann-geophys.net/11/717/1993/.

Shue, J.-H., Newell, P., Liou, K., and Meng, C.-I.: The quantitative relationship between auroral brightness and solar EUV Pedersen conductance, J. Geophys. Res., 106, 5883-5894, 2001.

Solomon, S.: Auroral electron transport using the Monte Carlo method, Geophys. Res. Lett., 20, 185-189, 1993.

Strickland, D., Book, D., Coffey, T., and Fedder, J.: Transport equation technique for the deposition of auroral electrons, J. Geophys. Res., 81, 2755-2764, 1976.

Sugiura, M.: A fundamental magnetosphere-ionosphere coupling mode involving field-aligned currents as deduced from DE 2 observations, Geophys. Res. Lett., 11, 877-880, 1984.

Temerin, M. and Carlson, C.: Current-voltage relationship in the downward auroral current region, Geophys. Res. Lett., 25, 2365 2368, 1998.

Trakhtengertz, V. and Feldstein, A.: Quiet auroral arcs: ionospheric effects of magnetospheric convection stratification, Planet. Space Sci., 32, 127-134, 1984

Vedin, J. and Rönnmark, K.: Electrostatic potentials in the downward auroral current region, J. Geophys. Res., 110, A08 207, 2005.

Vo, H. and Murphree, J.: A study of dayside auroral bright spots seen by the Viking auroral imager, J. Geophys. Res., 100, 3649 3655, 1995.

Vogt, J., Frey, H. U., Haerendel, G., Höfner, H., and Semeter, J. L.: Shear velocity profiles associated with auroral curls, J. Geophys. Res., 104, 17 277-17 288, 1999.

Wei, C., Lee, L. C., and La Belle-Hamer, A.: A simulation study of vortex structure in the low-latitude boundary layer, J. Geophys. Res., 95, 20 793-20 807, 1990.

Wei, C., Sonnerup, B., and Lotko, W.: Model of the low-latitude boundary layer with finite field-aligned potential drops and nonconstant mapping factors, J. Geophys. Res., 101, 21 463-21 479, 1996.

Yamamoto, T. and Ozaki, M.: A numerical model of the dayside aurora, J. Geophys. Res. (Space Physics), 110, 5215, doi: 10.1029/2004JA010786, 2005. 